\title{
POSITIVE STATIONARY SOLUTIONS AND SPREADING SPEEDS OF KPP EQUATIONS IN LOCALLY SPATIALLY INHOMOGENEOUS MEDIA*
}

\author{
LIANG KONG ${ }^{\dagger}$ AND WENXIAN SHEN ${ }^{\dagger}$
}

\begin{abstract}
The current paper is concerned with positive stationary solutions and spatial spreading speeds of KPP type evolution equations with local (i.e. the standard Laplacian) or nonlocal or discrete dispersal in locally spatially inhomogeneous media. It is shown that such an equation has a unique globally stable positive stationary solution and has a spreading speed in every direction. Moreover, it is shown that the localized spatial inhomogeneity of the medium neither slows down nor speeds up the spatial spreading in all the directions.

Key words. KPP equations, random dispersal, nonlocal dispersal, discrete dispersal, localized spatial inhomogeneity, spreading speed, positive stationary solution, principal eigenvalue, subsolution, super-solution, comparison principle.
\end{abstract}

AMS subject classifications. 35K57, 45G10, 58D20, 92D25.

1. Introduction. The current paper is devoted to the study of spatial spreading dynamics of species in locally spatially inhomogeneous environments or media. Reaction diffusion equations of the form

$$
u_{t}(t, x)=\Delta u(t, x)+u(t, x) f_{1}(x, u(t, x)), \quad x \in \mathbb{R}^{N}
$$

are widely used to model the population dynamics of many species in unbounded environments, where $u(t, x)$ is the population density of the species at time $t$ and location $x, \Delta u$ characterizes the internal interaction of the organisms, and $f_{1}(x, u)$ represents the growth rate of the population, which satisfies that $f_{1}(x, u)<0$ for $u \gg 1$ and $\partial_{u} f_{1}(x, u)<0$ for $u \geq 0$ (see [1], [2], [9], [22], [24], [25], [40], [51], [67], [69], [71], [72], [76], etc.).

When using (1.1) to model the population dynamics of a species, it is assumed that the underlying environment is not patchy and the internal interaction of the organisms is random and local (i.e. the organisms move randomly between the adjacent spatial locations). In practice, the environments in which many species live may be patchy and/or the internal interaction of the organisms may be nonlocal. To model the population dynamics of a species in the case that the underlying environment is not patchy but the internal interaction is nonlocal, the following nonlocal dispersal equation is often used,

$$
u_{t}(t, x)=\int_{\mathbb{R}^{N}} \kappa(y-x) u(t, y) d y-u(t, x)+u(t, x) f_{2}(x, u(t, x)), \quad x \in \mathbb{R}^{N},
$$

where $\kappa(\cdot)$ is a smooth convolution kernel supported on a ball centered at the origin (that is, there is a $\delta_{0}>0$ such that $\kappa(z)>0$ if $\|z\|<\delta_{0}, \kappa(z)=0$ if $\|z\| \geq \delta_{0}$, where $\|\cdot\|$ denotes the norm in $\mathbb{R}^{N}$ and $\delta_{0}$ represents the nonlocal dispersal distance), $\int_{\mathbb{R}^{N}} \kappa(z) d z=1$, and $f_{2}(\cdot, \cdot)$ is of the same property as $f_{1}$ in (1.1) (see [3], [10], [16], $[17],[18],[23],[27],[37],[39],[41],[42]$, etc.). Spatially discrete dispersal equations of

\footnotetext{
*Received June 24, 2011; accepted for publication June 18, 2012. Partially supported by NSF grant DMS-0907752.

$\dagger$ Department of Mathematics and Statistics, Auburn University, Auburn University, AL 36849, USA (\{lzk0002; wenxish $\} @$ auburn.edu).
} 
the following form arise when modeling the population dynamics of species living in patchy environments,

$$
u_{t}(t, j)=\sum_{k \in K} a_{k}(u(t, j+k)-u(t, j))+u(t, j) f_{3}(j, u(t, j)), \quad j \in \mathbb{Z}^{N}
$$

where $K=\left\{k \in \mathbb{Z}^{N} \mid\|k\|=1\right\}, a_{k}(k \in K)$ are positive constants, and $f_{3}(j, u)<0$ for $u \gg 1$ and $\partial_{u} f_{3}(j, u)<0$ for $u \geq 0$ (see [22], [48], [51], [67], [68], [71], [72], [73], etc.).

Spatial spreading dynamics is one of the central dynamical issues of (1.1)-(1.3). Roughly speaking, it is about how fast the population spreads as time evolves. E.g., letting $\mathcal{H}=\mathbb{R}^{N}$ in the case (1.1) and (1.2) and $\mathcal{H}=\mathbb{Z}^{N}$ in the case of (1.3), $\xi \in$ $S^{N-1}:=\left\{\xi \in \mathbb{R}^{N} \mid\|\xi\|=1\right\}$, and a given initial population $u_{0}$ satisfy for some $\sigma_{0}>0$ that $u_{0}(x) \geq \sigma_{0}$ for $x \in \mathcal{H}$ with $x \cdot \xi \ll-1$ and $u_{0}(x)=0$ for $x \in \mathcal{H}$ with $x \cdot \xi \gg 1$ $(x \cdot \xi$ is the inner product of $x$ and $\xi)$, how fast does the population invade into the region with no population initially?

Since the pioneering works by Fisher [25] and Kolmogorov, Petrowsky, Piscunov [40] on the following special case of (1.1)

$$
u_{t}(t, x)=u_{x x}(t, x)+u(t, x)(1-u(t, x)), \quad x \in \mathbb{R},
$$

a vast amount research has been carried out toward the spatial spreading dynamics of (1.1)-(1.3) with $f_{i}(\cdot, \cdot)(i=1,2,3)$ being independent of the space variable or periodic in the space variable, which reflects the spatial periodicity of the media. See, for example, [1], [2], [6], [38], [44], [45], [46], [58], [70], [71], etc. for the study of (1.1) in the case that $f_{1}(x, u)$ is independent of $x$ and see [5], [7], [26], [31], [35], [52], [54], [55], [72], etc. for the study of $(1.1)$ in the case that $f_{1}(x, u)$ is periodic in $x$; see [20], [21], [43], etc. for the study of $(1.2)$ in the case that $f_{2}(x, u)$ is independent of $x$ and see [33], [64], [65], [66], etc. for the study of $(1.2)$ in the case that $f_{2}(x, u)$ is periodic in $x$; and see [11], [12], [13], [36], [47], [71], [74], etc. for the study of (1.3) in the case that $f_{3}(j, u)$ is independent of $j$ and [28], [29], [30], [72], etc. for the study of $(1.3)$ in the case that $f_{3}(j, u)$ is periodic in $j$. In such cases, the spatial spreading dynamics is quite well understood. For example, consider (1.1) and assume that $f_{1}\left(x+p_{i} \mathbf{e}_{\mathbf{i}}, u\right)=f_{1}(x, u)$ for $i=1,2, \cdots, N$, where $p_{i}(i=1,2, \cdots, N)$ are positive constants and

$$
\mathbf{e}_{\mathbf{i}}=\left(\delta_{i 1}, \delta_{i 2}, \cdots, \delta_{i N}\right), \delta_{i j}=1 \text { if } i=j \text { and } 0 \text { if } i \neq j .
$$

If the principal eigenvalue of the following eigenvalue problem associated to the linearized equation of (1.1) at $u=0$,

$$
\left\{\begin{array}{l}
\Delta u(x)+f_{1}(x, 0) u(x)=\lambda u(x), \quad x \in \mathbb{R}^{N} \\
u\left(x+p_{i} \mathbf{e}_{\mathbf{i}}\right)=u(x), \quad x \in \mathbb{R}^{N},
\end{array}\right.
$$

is positive, then (1.1) has a unique positive stationary solution $u_{1}^{*}(\cdot)$ with $u_{1}^{*}\left(\cdot+p_{i} \mathbf{e}_{\mathbf{i}}\right)=$ $u_{1}^{*}(\cdot)$ and for any $\xi \in S^{N-1}:=\left\{\xi \in \mathbb{R}^{N} \mid\|\xi\|=1\right\}$, (1.1) has a positive spreading speed $c_{1}^{*}(\xi)$ in the direction of $\xi$ in the following sense (see Definition 2.1 for detail): for any given bounded $u_{0} \in C\left(\mathbb{R}^{N}, \mathbb{R}^{+}\right)$with $\liminf _{x \cdot \xi \rightarrow-\infty} u_{0}(x)>0$ and $u_{0}(x)=0$ for $x \cdot \xi \gg 1$,

$$
\liminf _{t \rightarrow \infty} \inf _{x \cdot \xi \leq c t} u_{1}\left(t, x ; u_{0}\right)>0 \quad \forall c<c_{1}^{*}(\xi)
$$


and

$$
\limsup _{t \rightarrow \infty} \sup _{x \cdot \xi \geq c t} u_{1}\left(t, x ; u_{0}\right)=0 \quad \forall c>c_{1}^{*}(\xi)
$$

where $u_{1}\left(t, x ; u_{0}\right)$ denotes the solution of $(1.1)$ with $u_{1}\left(0, x ; u_{0}\right)=u_{0}(x)$. Observe that (1.1) has also traveling wave solutions which connect $u_{1}^{*}(\cdot)$ and 0 and propagate in the direction of $\xi$ with speeds greater than or equal $c_{1}^{*}(\xi)$ and there is no such traveling wave solution of slower speed (see [7], [45], [59], [72] for the definition of spatially periodic traveling wave solutions). Hence $c_{1}^{*}(\xi)$ is also the minimal wave speed of traveling wave solutions propagating in the direction of $\xi$. See [7], [35], [45], [72] for the above mentioned results for (1.1) and see [64], [65], [66] for similar results for (1.2) and [29], [30], [36], [45], [72], [74] for similar results for (1.3).

In the current paper, we consider (1.1)-(1.3) in the case that the growth rates depend on the space variable, but only when it is in some bounded subset of the underlying media, which reflects the localized spatial inhomogeneity of the media. More precisely, let

$$
\left\{\begin{array}{l}
\mathcal{H}_{1}=\mathcal{H}_{2}=\mathbb{R}^{N} \\
\mathcal{H}_{3}=\mathbb{Z}^{N}
\end{array}\right.
$$

We assume

(H1) $f_{i}: \mathcal{H}_{i} \times \mathbb{R} \rightarrow \mathbb{R}$ is a $C^{2}$ function, $f_{i}(x, u)<0$ for all $(x, u) \in \mathcal{H}_{i} \times \mathbb{R}^{+}$with $u \geq \beta_{0}$ for some $\beta_{0}>0$, and $\partial_{u} f_{i}(x, u)<0$ for all $(x, u) \in \mathcal{H}_{i} \times \mathbb{R}^{+}$, where $i=1,2,3$.

(H2) $f_{i}(x, u)=f_{i}^{0}(u)$ for some $C^{2}$ function $f_{i}^{0}: \mathbb{R} \rightarrow \mathbb{R}$ and all $(x, u) \in \mathcal{H}_{i} \times \mathbb{R}$ with $\|x\| \geq L_{0}$ for some $L_{0}>0$, and $f_{i}^{0}(0)>0$, where $i=1,2,3$.

Assume (H1) and (H2). Then (1.1), (1.2), and (1.3) have the following limit equations as $\|x\| \rightarrow \infty$ or $\|j\| \rightarrow \infty$,

$$
\begin{gathered}
u_{t}(t, x)=\Delta u(t, x)+u(t, x) f_{1}^{0}(u(t, x)), \quad x \in \mathbb{R}^{N}, \\
u_{t}(t, x)=\int_{\mathbb{R}^{N}} \kappa(y-x) u(t, y) d y-u(t, x)+u(t, x) f_{2}^{0}(u(t, x)), \quad x \in \mathbb{R}^{N},
\end{gathered}
$$

and

$$
u_{t}(t, j)=\sum_{k \in K} a_{k}(u(t, j+k)-u(t, j))+u(t, j) f_{3}^{0}(u(t, j)), \quad j \in \mathbb{Z}^{N} .
$$

Equations (1.7), (1.8), and (1.9) will play an important role in the study of (1.1), (1.2), and (1.3). Clearly, (1.7) has similar spatial spreading dynamics as that of (1.4), that is, it has a unique positive constant solution $u_{1}^{0}$ and has a spatial spreading speed $c_{1}^{0}(\xi)$ in the direction of $\xi$ for every $\xi \in S^{N-1}$. Equations (1.8) (resp. (1.9)) has similar properties as that of (1.7), that is, (1.8) (resp. (1.9)) has a unique positive constant stationary solution $u_{2}^{0}\left(\right.$ resp. $\left.u_{3}^{0}\right)$ and has a spatial spreading speed $c_{2}^{0}(\xi)\left(\operatorname{resp} . c_{3}^{0}(\xi)\right)$ in the direction of $\xi$ for every $\xi \in S^{N-1}$ (see Definition 2.1 for detail).

Our objective is to explore the spatial spreading dynamics of (1.1)-(1.3) with localized spatial inhomogeneity. The main results of this paper can be summarized as follows:

- Assume (H1) and (H2). Then (1.1) (resp. (1.2), (1.3)) has a unique positive stationary solution $u_{1}^{*} \in C\left(\mathbb{R}^{N}, \mathbb{R}^{+}\right)$(resp. $u_{2}^{*} \in C\left(\mathbb{R}^{N}, \mathbb{R}^{+}\right), u_{3}^{*} \in C\left(\mathbb{Z}^{N}, \mathbb{R}^{+}\right)$) 
satisfying that $\inf _{x \in \mathbb{R}^{N}} u_{1}^{*}(x)>0$ (resp. $\inf _{x \in \mathbb{R}^{N}} u_{2}^{*}(x)>0, \inf _{j \in \mathbb{Z}^{N}} u_{3}^{*}(j)$ ) and $\lim _{\|x\| \rightarrow \infty} u_{1}^{*}(x)=u_{1}^{0}$ (resp. $\lim _{\|x\| \rightarrow \infty} u_{2}^{*}(x)=u_{2}^{0}, \lim _{\|j\| \rightarrow \infty} u_{3}^{*}(j)=u_{3}^{0}$ ). Moreover, $u=u_{i}^{*}(\cdot)$ is globally asymptotically stable with respect to (strongly) positive perturbations (and hence $u \equiv 0$ is an unstable stationary solution of $(1 . i))(i=1,2,3)$ (see Theorem 2.1).

- Assume (H1) and (H2). Then (1.1) (resp. (1.2), (1.3)) has a positive spatial spreading speed $c_{1}^{*}(\xi)$ (resp. $\left.c_{2}^{*}(\xi), c_{3}^{*}(\xi)\right)$ in the direction of $\xi$ for every $\xi \in S^{N-1}$ (see Definition 2.1 for the definition of spreading speeds). Moreover, $c_{1}^{*}(\xi)=c_{1}^{0}(\xi)$ (resp. $\left.c_{2}^{*}(\xi)=c_{2}^{0}(\xi), c_{3}^{*}(\xi)=c_{3}^{0}(\xi)\right)$ for all $\xi \in S^{N-1}$, where $c_{1}^{0}(\xi)$ (resp. $\left.c_{2}^{0}(\xi), c_{3}^{0}(\xi)\right)$ is the spatial spreading speed of (1.7) (resp. (1.8), (1.9)) in the direction of $\xi$ (see Theorem $2.2)$.

- Assume (H1) and (H2). Then the solution of (1.1) (resp. (1.2), (1.3)) with a nonnegative initial data which has a nonempty compact set spreads neither slower than $\inf \left\{c_{1}^{*}(\xi) \mid \xi \in S^{N-1}\right\}\left(\right.$ resp $\left.\inf \left\{c_{2}^{*}(\xi) \mid \xi \in S^{N-1}\right\}, \inf \left\{c_{3}^{*}(\xi) \mid \xi \in S^{N-1}\right\}\right)$ nor faster than $\sup \left\{c_{1}^{*}(\xi) \mid \xi \in S^{N-1}\right\}$ (resp $\sup \left\{c_{2}^{*}(\xi) \mid \xi \in S^{N-1}\right\}$, $\left.\sup \left\{c_{3}^{*}(\xi) \mid \xi \in S^{N-1}\right\}\right)$ (see Theorem 2.3 for detail).

The above results reveal such an important biological scenario: the localized spatial inhomogeneity of the media does not prevent the population to persist and to spread, moreover, it neither slows down nor speeds up the spatial spread of the population.

It should be pointed out that the authors of [56] considered the transition fronts, which are generalizations of traveling wave solutions, of (1.1) in the case that $N=1$, $f(x, 1)=0$, and $f(x, 0)>0$. They provided conditions under which transition fronts of (1.1) exist and also showed that (1.1) may not have transition fronts. Hence the localized spatial inhomogeneity of the media may prevent the existence of transition fronts.

We remark that in literature (1.1) (resp. (1.2), (1.3)) with $f_{1}(x, u)$ (resp. $f_{2}(x, u)$, $\left.f_{3}(j, u)\right)$ being decreasing in $u$ and negative for $u \gg 1$ and $u \equiv 0$ being an unstable solution is called a Fisher type or KPP type or monostable equation. The reader is referred to [4], [56], and references therein for the study of transition solutions of general spatially inhomogeneous Fisher or KPP type equations and to [34], [60]-[63] for the study of spatial spreading dynamics of general temporally inhomogeneous Fisher or KPP type equations. The reader is also referred to [49], [50], and references therein for the study of transition solutions of general spatially inhomogeneous ignition type equations.

We also remark that it would be interesting to study the spatial spreading dynamics of KPP type equations in inhomogeneous media with more general limit media, say, equation $(1 . \mathrm{i})(i=1,2,3)$ with $f_{i}(x, u)$ being replaced by $f_{i}(t, x, u)$ satisfying that $f_{i}(t, x, u)-f_{i}^{0}(t, x, u) \rightarrow 0$ as $\|x\| \rightarrow \infty$ for some function $f_{i}^{0}(t, x, u)$ which is periodic in $t$ and/or $x$. We will consider such general case elsewhere.

The rest of the paper is organized as follows. In section 2, we introduce the standing notions to be used in the paper and the definition of spreading speeds and state the main results of the paper (i.e. Theorems $2.1,2.2$, and 2.3). In section 3, we present some preliminary materials to be used in later sections. Section 4 is devoted to the study of positive stationary solutions of (1.1)-(1.3). Theorem 2.1 is proved in this section. In section 5, we explore the existence of spreading speeds of (1.1)-(1.3) and prove Theorems 2.2 and 2.3 . 
Acknowledgment. The authors thank the referee for careful reading of the current paper and valuable comments which led to the improvement of our original manuscript.

2. Standing notions, definitions, and main results. In this section, we first introduce some standing notations and the definition of spreading speeds. We then state the main results of the paper.

Let $\mathcal{H}_{i}$ be as in (1.6). Let $p=\left(p_{1}, p_{2}, \cdots, p_{N}\right)$ with $p_{i}>0$ for $i=1,2, \cdots, N$. We define the Banach spaces $X_{i, p}(i=1,2)$ by

$$
X_{1, p}=\left\{u \in C\left(\mathbb{R}^{N}, \mathbb{R}\right) \mid u\left(\cdot+p_{i} \mathbf{e}_{\mathbf{i}}\right)=u(\cdot), \quad i=1, \ldots, N\right\}
$$

with norm $\|u\|_{X_{1, p}}=\max _{x \in \mathbb{R}^{N}}|u(x)|$, and

$$
X_{2, p}=X_{1, p}
$$

(the introduction of $X_{2, p}$ is for the convenience in notation). If $p_{i} \in \mathbb{N}$, we define $X_{3, p}$ by

$$
X_{3, p}=\left\{u \in C\left(\mathbb{Z}^{N}, \mathbb{R}\right) \mid u\left(\cdot+p_{i} \mathbf{e}_{\mathbf{i}}\right)=u(\cdot), \quad i=1,2, \cdots, N\right\}
$$

with norm $\|u\|_{X_{3, p}}=\max _{j \in \mathbb{Z}^{N}}|u(j)|$. Let

$$
X_{i, p}^{+}=\left\{u \in X_{i, p} \mid u(x) \geq 0 \forall x \in \mathcal{H}_{i}\right\}
$$

and

$$
X_{i, p}^{++}=\left\{u \in X_{i, p} \mid u(x)>0 \forall x \in \mathcal{H}_{i}\right\}
$$

for $i=1,2,3$. We define $X_{i}(i=1,2,3)$ by

$$
X_{1}=\left\{u \in C\left(\mathbb{R}^{N}, \mathbb{R}\right) \mid u \text { is uniformly continuous and bounded }\right\}
$$

with norm $\|u\|_{X_{1}}=\sup _{x \in \mathbb{R}^{N}}|u(x)|$,

$$
X_{2}=X_{1}
$$

(again the introduction of $X_{2}$ is for the convenience in notation), and

$$
X_{3}=\left\{u \in C\left(\mathbb{Z}^{N}, \mathbb{R}\right) \mid u \text { is bounded }\right\}
$$

with norm $\|u\|_{X_{3}}=\sup _{j \in \mathbb{Z}^{N}}|u(j)|$. Let

$$
X_{i}^{+}=\left\{u \in X_{i} \mid u(x) \geq 0 \forall x \in \mathcal{H}_{i}\right\}
$$

and

$$
X_{i}^{++}=\left\{u \in X_{i}^{+} \mid \inf _{x \in \mathcal{H}_{i}} u(x)>0\right\}
$$

for $i=1,2,3$.

If no confusion occurs, we may write $\|\cdot\|_{X_{i, p}}$ and $\|\cdot\|_{X_{i}}$ as $\|\cdot\|(i=1,2,3)$.

Assume (H1). By general semigroup theory (see [32], [57]), for any $u_{0} \in X_{1}$ (resp. $\left.u_{0} \in X_{2}, u_{0} \in X_{3}\right),(1.1)$ (resp. (1.2), (1.3)) has a unique local solution $u_{1}\left(t, \cdot ; u_{0}\right)$ (resp. $\left.u_{2}\left(t, \cdot ; u_{0}\right), u_{3}\left(t, \cdot ; u_{0}\right)\right)$ with $u_{1}\left(0, \cdot ; u_{0}\right)=u_{0}(\cdot)\left(\right.$ resp. $u_{2}\left(0, \cdot ; u_{0}\right)=u_{0}(\cdot)$, 
$\left.u_{3}\left(0, \cdot ; u_{0}\right)=u_{0}(\cdot)\right)$. Moreover, if $u_{0} \in X_{i}^{+}$, then $u_{i}\left(t, \cdot ; u_{0}\right)$ exist and $u_{i}\left(t, \cdot ; u_{0}\right) \in X_{i}^{+}$ for all $t \geq 0(i=1,2,3)$ (see Proposition 3.2).

Let

$$
S^{N-1}=\left\{\xi \in \mathbb{R}^{N} \mid\|\xi\|=1\right\}
$$

For given $\xi \in S^{N-1}$ and $u \in X_{i}^{+}$, we define

$$
\liminf _{x \cdot \xi \rightarrow-\infty} u(x)=\liminf _{r \rightarrow-\infty} \inf _{x \in \mathcal{H}_{i}, x \cdot \xi \leq r} u(x) .
$$

For given $u:[0, \infty) \times \mathcal{H}_{i} \rightarrow \mathbb{R}(1 \leq i \leq 3)$ and $c>0$, we define

$$
\begin{aligned}
& \liminf _{x \cdot \xi \leq c t, t \rightarrow \infty} u(t, x)=\liminf _{t \rightarrow \infty} \inf _{x \in \mathcal{H}_{i}, x \cdot \xi \leq c t} u(t, x), \\
& \limsup _{x \cdot \xi \geq c t, t \rightarrow \infty} u(t, x)=\limsup _{t \rightarrow \infty} \sup _{x \in \mathcal{H}_{i}, x \cdot \xi \geq c t} u(t, x) .
\end{aligned}
$$

The notions $\limsup _{|x \cdot \xi| \leq c t, t \rightarrow \infty} u(t, x), \quad \limsup _{|x \cdot \xi| \geq c t, t \rightarrow \infty} u(t, x), \limsup _{\|x\| \leq c t, t \rightarrow \infty} u(t, x), \quad$ and $\limsup _{\| x} u(t, x)$ are defined similarly. We define $X_{i}^{+}(\xi)(i=1,2,3)$ by $\|x\| \geq c t, t \rightarrow \infty$

$$
X_{i}^{+}(\xi)=\left\{u \in X_{i}^{+} \mid \liminf _{x \cdot \xi \rightarrow-\infty} u(x)>0, \quad u(x)=0 \text { for } x \cdot \xi \gg 1\right\} .
$$

Definition 2.1 (Spatial spreading speed). For given $\xi \in S^{N-1}$ and given $i \in \mathbb{N}$ $(1 \leq i \leq 3)$, a real number $c_{i}^{*}(\xi)$ is called the spatial spreading speed of (1.i) in the direction of $\xi$ if for any $u_{0} \in X_{i}^{+}(\xi)$,

$$
\liminf _{x \cdot \xi \leq c t, t \rightarrow \infty} u_{i}\left(t, x ; u_{0}\right)>0 \quad \forall c<c_{i}^{*}(\xi)
$$

and

$$
\limsup _{x \cdot \xi \geq c t, t \rightarrow \infty} u_{i}\left(t, x ; u_{0}\right)=0 \quad \forall c>c_{i}^{*}(\xi)
$$

The main results of this paper are stated in the following three theorems.

TheOrem 2.1 (Positive stationary solutions). Assume (H1) and (H2).

(1) (Existence) Equation (1.1) (resp. (1.2), (1.3)) has a unique stationary solution $u=u_{1}^{*}(\cdot) \in X_{1}^{++}\left(\right.$resp. $\left.u=u_{2}^{*}(\cdot) \in X_{2}^{++}, u=u_{3}^{*}(\cdot) \in X_{3}^{++}\right)$. Moreover,

$$
\lim _{r \rightarrow \infty} \sup _{x \in \mathcal{H}_{i},\|x\| \geq r}\left|u_{i}^{*}(x)-u_{i}^{0}\right|=0
$$

where $u_{i}^{0}>0$ is such that $f_{i}^{0}\left(u_{i}^{0}\right)=0$ and $i=1,2,3$.

(2) (Stability) For any $u_{0} \in X_{i}^{++}, \lim _{t \rightarrow \infty}\left\|u_{i}\left(t, \cdot ; u_{0}\right)-u_{i}^{*}(\cdot)\right\|_{X_{i}}=0$.

(3) (Stability) For any $u_{0} \in X_{i}^{+} \backslash\{0\}, \lim _{t \rightarrow \infty} u_{i}\left(t, x ; u_{0}\right)=u_{i}^{*}(x)$ uniformly in $x$ on bounded sets. 
Theorem 2.2 (Existence and characterization of spreading speeds). Assume (H1) and (H2). Then for any given $\xi \in S^{N-1}$, (1.1) (resp. (1.2), (1.3)) has a positive spreading speed $c_{1}^{*}(\xi)$ (resp. $\left.c_{2}^{*}(\xi), c_{3}^{*}(\xi)\right)$ in the direction of $\xi$. Moreover, for any $u_{0} \in X_{i}^{+}(\xi)$,

$$
\liminf _{x \cdot \xi \leq c t, t \rightarrow \infty}\left|u_{i}\left(t, x ; u_{0}\right)-u_{i}^{*}(x)\right|=0 \quad \forall c<c_{i}^{*}(\xi),
$$

and

$$
c_{i}^{*}(\xi)=c_{i}^{0}(\xi) \quad \text { for } \quad i=1,2,3,
$$

where

$$
\begin{gathered}
c_{1}^{0}(\xi)=\inf _{\mu>0} \frac{f_{1}^{0}(0)+\mu^{2}}{\mu}=2 \sqrt{f_{1}^{0}(0)}, \\
c_{2}^{0}(\xi)=\inf _{\mu>0} \frac{\int_{\mathbb{R}^{N}} e^{-\mu z \cdot \xi} \kappa(z) d z-1+f_{2}^{0}(0)}{\mu},
\end{gathered}
$$

and

$$
c_{3}^{0}(\xi)=\inf _{\mu>0} \frac{\sum_{k \in K} a_{k}\left(e^{-\mu k \cdot \xi}-1\right)+f_{3}^{0}(0)}{\mu}
$$

are the spatial spreading speeds of (1.7), (1.8), and (1.9) in the direction of $\xi$, respectively.

TheOREM 2.3 (Spreading features of spreading speeds). Assume (H1) and (H2) and $1 \leq i \leq 3$. Then for any given $\xi \in S^{N-1}$, the following hold.

(1) For each $u_{0} \in X_{i}^{+}$satisfying that $u_{0}(x)=0$ for $x \in \mathcal{H}_{i}$ with $|x \cdot \xi| \gg 1$,

$$
\limsup _{|x \cdot \xi| \geq c t, t \rightarrow \infty} u_{i}\left(t, x ; u_{0}\right)=0 \quad \forall c>\max \left\{c_{i}^{*}(\xi), c_{i}^{*}(-\xi)\right\} .
$$

(2) For each $\sigma>0, r>0$, and $u_{0} \in X_{i}^{+}$satisfying that $u_{0}(x) \geq \sigma$ for $x \in \mathcal{H}_{i}$ with $|x \cdot \xi| \leq r$,

$$
\limsup _{|x \cdot \xi| \leq c t, t \rightarrow \infty}\left|u_{i}\left(t, x ; u_{0}\right)-u_{i}^{*}(x)\right|=0 \quad \forall 0<c<\min \left\{c_{i}^{*}(\xi), c_{i}^{*}(-\xi)\right\} .
$$

(3) For each $u_{0} \in X_{i}^{+}$satisfying that $u_{0}(x)=0$ for $x \in \mathcal{H}_{i}$ with $\|x\| \gg 1$,

$$
\limsup _{\|x\| \geq c t, t \rightarrow \infty} u_{i}\left(t, x ; u_{0}\right)=0 \quad \forall c>\sup _{\xi \in S^{N-1}} c_{i}^{*}(\xi) .
$$

(4) For each $\sigma>0, r>0$, and $u_{0} \in X_{i}^{+}$satisfying that $u_{0}(x) \geq \sigma$ for $\|x\| \leq r$,

$$
\limsup _{\|x\| \leq c t, t \rightarrow \infty}\left|u_{i}\left(t, x ; u_{0}\right)-u_{i}^{*}(x)\right|=0 \quad \forall 0<c<\inf _{\xi \in S^{N-1}} c_{i}^{*}(\xi) .
$$

To indicate the dependence of $u_{i}^{*}(\cdot)$ and $c_{i}^{*}(\xi)$ on $f_{i}$, we may sometime write $u_{i}^{*}(\cdot)$ and $c_{i}^{*}(\xi)$ as $u_{i}^{*}\left(\cdot ; f_{i}(\cdot, \cdot)\right)$ and $c_{i}^{*}\left(\xi ; f_{i}(\cdot, \cdot)\right)$, respectively.

We remark that most parts of Theorem 2.1 for (1.1) can de deduced from [8]. In the current paper, we develop a unified approach to deal with the existence, uniqueness, and stability of positive stationary solutions and the existence and characterization of spatial spreading speeds for KPP equations with three different types of dispersal strategies in locally spatially inhomogeneous media. 
3. Preliminary. In this section, we present some preliminary materials to be used in later sections, including some basic properties of solutions of (1.1)-(1.3); principal eigenvalue theories for spatially periodic dispersal operators with random, nonlocal, and discrete dispersals; and spatial spreading dynamics of KPP equations in spatially periodic media.

3.1. Basic properties of KPP equations. In this subsection, we present some basic properties of solutions of (1.1)-(1.3), including comparison principle, global existence, convergence in open compact topology, and decreasing of the so called part metric along the solutions. Throughout this subsection, we assume (H1).

Let $X_{1}, X_{2}$, and $X_{3}$ be as in (2.6), (2.7), and (2.8), respectively. For given $u_{0} \in X_{1}$ (resp. $\left.u_{0} \in X_{2}, u_{0} \in X_{3}\right)$, let $u_{1}\left(t, \cdot ; u_{0}\right)$ (resp. $\left.u_{2}\left(t, \cdot ; u_{0}\right), u_{3}\left(t, \cdot ; u_{0}\right)\right)$ be the (local) solution of $(1.1)(\operatorname{resp} .(1.2),(1.3))$ with $u_{1}\left(0, \cdot ; u_{0}\right)=u_{0}(\cdot)\left(\operatorname{resp} . u_{2}\left(0, \cdot ; u_{0}\right)=u_{0}(\cdot)\right.$, $\left.u_{3}\left(0, \cdot ; u_{0}\right)=u_{0}(\cdot)\right)$.

Let $X_{i}^{+}$and $X_{i}^{++}(i=1,2,3)$ be as in (2.9) and (2.10). For given $1 \leq i \leq 3$ and $u, v \in X_{i}$, we define

$$
u \leq v(u \geq v) \text { if } v-u \in X_{i}^{+}\left(u-v \in X_{i}^{+}\right)
$$

and

$$
u \ll v(u \gg v) \text { if } v-u \in X_{i}^{++}\left(u-v \in X_{i}^{++}\right) .
$$

For given continuous and bounded function $u:[0, T) \times \mathbb{R}^{N} \rightarrow \mathbb{R}$, it is called a super-solution (sub-solution) of (1.1) on $[0, T)$ if

$$
u_{t}(t, x) \geq(\leq) \Delta u(t, x)+u(t, x) f_{1}(x, u(t, x)) \quad \forall(t, x) \in(0, T) \times \mathbb{R}^{N} .
$$

Super-solutions (sub-solutions) of (1.2) and (1.3) are defined similarly.

Proposition 3.1 (Comparison principle). Assume (H1).

(1) Suppose that $u^{1}(t, x)$ and $u^{2}(t, x)$ are sub- and super-solutions of (1.1) (resp. $(1.2)$, (1.3)) on $[0, T)$ with $u^{1}(0, \cdot) \leq u^{2}(0, \cdot)$. Then $u^{1}(t, \cdot) \leq u^{2}(t, \cdot)$ for $t \in(0, T)$. Moreover, if $u^{1}(0, \cdot) \neq u^{2}(0, \cdot)$, then $u^{1}(t, x)<u^{2}(t, x)$ for $x \in \mathcal{H}_{1}$ (resp. $\left.x \in \mathcal{H}_{2}, x \in \mathcal{H}_{3}\right)$ and $t \in(0, T)$.

(2) If $u_{01}, u_{02} \in X_{i}$ and $u_{01} \leq u_{02}(1 \leq i \leq 3)$, then $u_{i}\left(t, \cdot ; u_{01}\right) \leq u_{i}\left(t, \cdot ; u_{02}\right)$ for $t>0$ at which both $u_{i}\left(t, \cdot ; u_{01}\right)$ and $u_{i}\left(t, \cdot ; u_{02}\right)$ exist.

(3) If $u_{01}, u_{02} \in X_{i}$ and $u_{01} \leq u_{02}, u_{01} \neq u_{02}(1 \leq i \leq 3)$, then $u_{i}\left(t, x ; u_{01}\right)<$ $u_{i}\left(t, x ; u_{02}\right)$ for all $x \in \mathcal{H}_{i}$ and $t>0$ at which both $u_{i}\left(t, \cdot ; u_{01}\right)$ and $u_{i}\left(t, \cdot ; u_{02}\right)$ exist.

(4) If $u_{01}, u_{02} \in X_{i}$ and $u_{01} \ll u_{02}(1 \leq i \leq 3)$, then $u_{i}\left(t, \cdot ; u_{01}\right) \ll u_{i}\left(t, \cdot ; u_{02}\right)$ for $t>0$ at which both $u_{i}\left(t, \cdot ; u_{01}\right)$ and $u_{i}\left(t, \cdot ; u_{02}\right)$ exist.

Proof. (1) The case $i=1$ follows from comparison principle for parabolic equations. The case $i=2$ follows from [64, Propositions 2.1 and 2.2]. The case $i=3$ follows from comparison principle for lattice differential equations (see the arguments in [14, Lemma 1]).

(2) and (3) follow from (1).

(4) We provide a proof for the case $i=2$. Other cases can be proved similarly. Take any $T>0$ such that both $u_{2}\left(t, \cdot ; u_{01}\right)$ and $u_{2}\left(t, \cdot ; u_{02}\right)$ exist on $[0, T]$. It suffices to prove that $u_{2}\left(t, \cdot ; u_{02}\right) \gg u_{2}\left(t, \cdot ; u_{01}\right)$ for $t \in[0, T]$. To this end, let $w(t, x)=$ $u_{2}\left(t, x ; u_{02}\right)-u_{2}\left(t, x ; u_{01}\right)$. Then $w(t, x)$ satisfies the following equation,

$$
w_{t}(t, x)=\int_{\mathbb{R}^{N}} \kappa(y-x) w(t, y) d y-w(t, x)+a(t, x) w(t, x),
$$


where

$$
\begin{aligned}
a(t, x)= & f_{2}\left(x, u_{2}\left(t, x ; u_{02}\right)\right) \\
& +u_{2}\left(t, x ; u_{01}\right) \int_{0}^{1} \partial_{u} f_{2}\left(x, s u_{2}\left(t, x ; u_{02}\right)+(1-s) u_{2}\left(t, x ; u_{01}\right)\right) d s .
\end{aligned}
$$

Let $M>0$ be such that $M \geq \sup _{x \in \mathbb{R}^{N}, t \in[0, T]}(1-a(t, x))$ and $\tilde{w}(t, x)=e^{M t} w(t, x)$. Then $\tilde{w}(t, x)$ satisfies

$$
\tilde{w}_{t}(t, x)=\int_{\mathbb{R}^{N}} \kappa(y-x) \tilde{w}(t, y) d y+[M-1+a(t, x)] \tilde{w}(t, x) .
$$

Let $\mathcal{K}: X_{2} \rightarrow X_{2}$ be defined by

$$
(\mathcal{K} u)(x)=\int_{\mathbb{R}^{N}} \kappa(y-x) u(y) d y \quad \text { for } \quad u \in X_{2} .
$$

Then $\mathcal{K}$ generates an analytic semigroup on $X_{2}$ and

$$
\tilde{w}(t, \cdot)=e^{\mathcal{K} t}\left(u_{02}-u_{01}\right)+\int_{0}^{t} e^{\mathcal{K}(t-\tau)}(M-1+a(\tau, \cdot)) \tilde{w}(\tau, \cdot) d \tau .
$$

Observe that $e^{\mathcal{K} t} u_{0} \geq 0$ for any $u_{0} \in X_{2}^{+}$and $t \geq 0$ and $e^{\mathcal{K} t} u_{0} \gg 0$ for any $u_{0} \in X_{2}^{++}$ and $t \geq 0$. Observe also that $u_{02}-u_{01} \in X_{2}^{++}$. By $(2), \tilde{w}(\tau, \cdot) \geq 0$ and hence $(M-1+a(\tau, \cdot)) \tilde{w}(\tau, \cdot) \geq 0$ for $\tau \in[0, T]$. It then follows that $\tilde{w}(t, \cdot) \gg 0$ and then $w(t, \cdot) \gg 0\left(\right.$ i.e. $\left.u_{2}\left(t, \cdot ; u_{02}\right) \gg u_{2}\left(t, \cdot ; u_{01}\right)\right)$ for $t \in[0, T]$.

Proposition 3.2 (Global existence). Assume (H1). For any given $1 \leq i \leq 3$ and $u_{0} \in X_{i}^{+}, u_{i}\left(t, \cdot ; u_{0}\right)$ exists for all $t \geq 0$.

Proof. Let $1 \leq i \leq 3$ and $u_{0} \in X_{i}^{+}$be given. There is $M \gg 1$ such that $0 \leq u_{0}(x) \leq M$ and $f_{i}(x, M)<0$ for all $x \in \mathcal{H}_{i}$. Then by Proposition 3.1,

$$
0 \leq u_{i}\left(t, \cdot ; u_{0}\right) \leq M
$$

for any $t>0$ at which $u_{i}\left(t, \cdot ; u_{0}\right)$ exists. It is then not difficult to prove that for any $T>0$ such that $u_{i}\left(t, \cdot ; u_{0}\right)$ exists on $(0, T), \lim _{t \rightarrow T} u_{i}\left(t, \cdot ; u_{0}\right)$ exists in $X_{i}$. This implies that $u_{i}\left(t, \cdot ; u_{0}\right)$ exists and $u_{i}\left(t, \cdot ; u_{0}\right) \geq 0$ for all $t \geq 0$.

For given $u, v \in X_{i}^{++}$, define

$$
\rho_{i}(u, v)=\inf \left\{\ln \alpha \mid \frac{1}{\alpha} u \leq v \leq \alpha u, \alpha \geq 1\right\} .
$$

Observe that $\rho_{i}(u, v)$ is well defined and there is $\alpha \geq 1$ such that $\rho_{i}(u, v)=\ln \alpha$. Moreover, $\rho_{i}(u, v)=\rho_{i}(v, u)$ and $\rho_{i}(u, v)=0$ iff $u \equiv v$. In literature, $\rho_{i}(u, v)$ is called the part metric between $u$ and $v$.

Proposition 3.3 (Decreasing of part metric). For given $1 \leq i \leq 3$ and $u_{0}, v_{0} \in$ $X_{i}^{++}$with $u_{0} \neq v_{0}, \rho_{i}\left(u_{i}\left(t, \cdot ; u_{0}\right), u_{i}\left(t, \cdot ; v_{0}\right)\right)$ is non-increasing in $t \in(0, \infty)$.

Proof. We give a proof for the case $i=1$. Other cases can be proved similarly.

First, note that there is $\alpha^{*}>1$ such that $\rho_{1}\left(u_{0}, v_{0}\right)=\ln \alpha^{*}$ and $\frac{1}{\alpha^{*}} u_{0} \leq v_{0} \leq$ $\alpha^{*} u_{0}$. By Proposition 3.1,

$$
u_{1}\left(t, \cdot ; v_{0}\right) \leq u_{1}\left(t, \cdot ; \alpha^{*} u_{0}\right) \quad \text { for } \quad t>0 .
$$


Let $v(t, x)=\alpha^{*} u_{1}\left(t, x ; u_{0}\right)$. Then

$$
\begin{aligned}
v_{t}(t, x) & =\Delta v(t, x)+v(t, x) f_{1}\left(x, u_{1}\left(t, x ; u_{0}\right)\right) \\
& =\Delta v(t, x)+v(t, x) f_{1}(x, v(t, x))+v(t, x) f_{1}\left(x, u_{1}\left(t, x ; u_{0}\right)\right)-v(t, x) f_{1}(x, v(t, x)) \\
& >\Delta v(t, x)+v(t, x) f_{1}(x, v(t, x)) .
\end{aligned}
$$

This together with Proposition 3.1 implies that

$$
u_{1}\left(t, \cdot ; \alpha^{*} u_{0}\right) \leq \alpha^{*} u_{1}\left(t, \cdot ; u_{0}\right) \quad \text { for } \quad t>0
$$

and then

$$
u_{1}\left(t, \cdot ; v_{0}\right) \leq \alpha^{*} u_{1}\left(t, \cdot ; u_{0}\right) \quad \text { for } \quad t>0
$$

Similarly, it can be proved that

$$
\frac{1}{\alpha^{*}} u_{1}\left(t, \cdot ; u_{0}\right) \leq u_{1}\left(t, \cdot ; v_{0}\right) \text { for } t>0 .
$$

It then follows that

$$
\rho_{1}\left(u_{1}\left(t, \cdot ; u_{0}\right), u_{1}\left(t, \cdot ; v_{0}\right)\right) \leq \rho_{1}\left(u_{0}, v_{0}\right) \quad \forall t>0
$$

and hence

$$
\rho_{1}\left(u_{1}\left(t_{2}, \cdot ; u_{0}\right), u_{1}\left(t_{2}, \cdot ; v_{0}\right)\right) \leq \rho_{1}\left(u_{1}\left(t_{1}, \cdot ; u_{0}\right), u_{1}\left(t_{1}, \cdot ; v_{0}\right)\right) \quad \forall 0 \leq t_{1}<t_{2} .
$$

To indicate the dependence of solutions of (1.1)-(1.3) on the nonlinearity, we may write $u_{i}\left(t, \cdot ; u_{0}\right)$ as $u_{i}\left(t, \cdot ; u_{0}, f_{i}(\cdot, \cdot)\right)$. Observe that for any $z_{n} \in \mathcal{H}_{i}$, if $\left\{z_{n}\right\}$ is a bounded sequence, then there are $z^{*} \in \mathcal{H}_{i}$ and $\left\{z_{n_{k}}\right\} \subset\left\{z_{n}\right\}$ such that $z_{n_{k}} \rightarrow z^{*}$ and $f_{i}\left(x+z_{n_{k}}, u\right) \rightarrow f_{i}\left(x+z^{*}, u\right)$ uniformly in $(x, u)$ on bounded sets. If $\left\{z_{n}\right\}$ is an unbounded sequence, then there is $z_{n_{k}}$ such that $f_{i}\left(x+z_{n_{k}}, u\right) \rightarrow f_{i}^{0}(u)$ uniformly in $(x, u)$ on bounded sets.

Proposition 3.4 (Convergence on compact subsets). Given $1 \leq i \leq 3$, suppose that $u_{0 n}, u_{0} \in X_{i}^{+}(n=1,2, \cdots),\left\{\left\|u_{0 n}\right\|\right\}$ is bounded, and $u_{0 n}(x) \rightarrow u_{0}(x)$ as $n \rightarrow \infty$ uniformly in $x$ on bounded sets.

(1) If $z_{n}, z^{*} \in \mathcal{H}_{i}(n=1,2, \cdots)$ are such that $f_{i}\left(x+z_{n}, u\right) \rightarrow f_{i}\left(x+z^{*}, u\right)$ as $n \rightarrow$ $\infty$ uniformly in $(x, u)$ on bounded sets, then for each $t>0, u_{i}\left(t, x ; u_{0 n}, f_{i}(\cdot+\right.$ $\left.\left.z_{n}, \cdot\right)\right) \rightarrow u_{i}\left(t, x ; u_{0}, f_{i}\left(\cdot+z^{*}, \cdot\right)\right)$ as $n \rightarrow \infty$ uniformly in $x$ on bounded sets.

(2) If $z_{n} \in \mathcal{H}_{i}(n=1,2, \cdots)$ are such that $f_{i}\left(x+z_{n}, u\right) \rightarrow f_{i}^{0}(u)$ as $n \rightarrow \infty$ uniformly in $(x, u)$ on bounded sets, then for each $t>0, u_{i}\left(t, x ; u_{0 n}, f_{i}(\cdot+\right.$ $\left.\left.z_{n}, \cdot\right)\right) \rightarrow u_{i}\left(t, x ; u_{0}, f_{i}^{0}(\cdot)\right)$ as $n \rightarrow \infty$ uniformly in $x$ on bounded sets.

Proof. We prove (1) with $i=2$. All other cases can be proved similarly.

Let $v^{n}(t, x)=u_{2}\left(t, x ; u_{0 n}, f_{2}\left(\cdot+z_{n}, \cdot\right)\right)-u_{2}\left(t, x ; u_{0}, f_{2}\left(\cdot+z^{*}, \cdot\right)\right)$. Then $v^{n}(t, x)$ satisfies

$$
v_{t}^{n}(t, x)=\int_{\mathbb{R}^{N}} \kappa(y-x) v^{n}(t, y) d y-v^{n}(t, x)+a_{n}(t, x) v^{n}(t, x)+b_{n}(t, x),
$$

where

$$
\begin{aligned}
a_{n}(t, x)= & f_{2}\left(x+z_{n}, u_{2}\left(t, x ; u_{0 n}, f_{2}\left(\cdot+z_{n}, \cdot\right)\right)\right)+u_{2}\left(t, x ; u_{0}, f_{2}\left(\cdot+z^{*}, \cdot\right)\right) \\
& \cdot \int_{0}^{1} \partial_{u} f_{2}\left(x+z_{n}, s u_{2}\left(t, x ; u_{0 n}, f_{2}\left(\cdot+z_{n}, \cdot\right)\right)+(1-s) u_{2}\left(t, x ; u_{0}, f_{2}\left(\cdot+z^{*}, \cdot\right)\right)\right) d s
\end{aligned}
$$


and

$$
\begin{aligned}
b_{n}(t, x)= & u_{2}\left(t, x ; u_{0}, f_{2}\left(\cdot+z^{*}, \cdot\right)\right) \\
& \cdot\left(f_{2}\left(x+z_{n}, u_{2}\left(t, x ; u_{0}, f_{2}\left(\cdot+z^{*}, \cdot\right)\right)\right)-f_{2}\left(x+z^{*}, u_{2}\left(t, x ; u_{0}, f_{2}\left(\cdot+z^{*}, \cdot\right)\right)\right)\right) .
\end{aligned}
$$

Observe that $\left\{a_{n}(t, x)\right\}$ is uniformly bounded and continuous in $t$ and $x$ and $b_{n}(t, x) \rightarrow$ 0 as $n \rightarrow \infty$ uniformly in $t \in[0, \infty)$ and $x$ on bounded sets.

Take a $\rho>0$. Let

$$
X_{2}(\rho)=\left\{u \in C\left(\mathbb{R}^{N}, \mathbb{R}\right) \mid u(\cdot) e^{-\rho\|\cdot\|} \in X_{2}\right\}
$$

with norm $\|u\|_{\rho}=\left\|u(\cdot) e^{-\rho\|\cdot\|}\right\|$. Note that $\mathcal{K}: X_{2}(\rho) \rightarrow X_{2}(\rho)$ also generates an analytic semigroup, where $\mathcal{K}$ is as in (3.3), and there are $M>0$ and $\omega>0$ such that

$$
\left\|e^{(\mathcal{K}-\mathcal{I}) t}\right\|_{X_{2}(\rho)} \leq M e^{\omega t} \quad \forall t \geq 0
$$

where $\mathcal{I}$ is the identity map on $X_{2}(\rho)$. Hence

$$
\begin{aligned}
v^{n}(t, \cdot)= & e^{(\mathcal{K}-\mathcal{I}) t} v^{n}(0, \cdot)+\int_{0}^{t} e^{(\mathcal{K}-\mathcal{I})(t-\tau)} a_{n}(\tau, \cdot) v^{n}(\tau, \cdot) d \tau \\
& +\int_{0}^{t} e^{(\mathcal{K}-\mathcal{I})(t-\tau)} b_{n}(\tau, \cdot) d \tau
\end{aligned}
$$

and then

$$
\begin{aligned}
\left\|v^{n}(t, \cdot)\right\|_{X_{2}(\rho)} \leq & M e^{\omega t}\left\|v^{n}(0, \cdot)\right\|_{X_{2}(\rho)}+M \sup _{\tau \in[0, t], x \in \mathbb{R}^{N}}\left|a_{n}(\tau, x)\right| \int_{0}^{t} e^{\omega(t-\tau)}\left\|v^{n}(\tau, \cdot)\right\|_{X_{2}(\rho)} d \tau \\
& +M \int_{0}^{t} e^{\omega(t-\tau)}\left\|b_{n}(\tau, \cdot)\right\|_{X_{2}(\rho)} d \tau \\
\leq & M e^{\omega t}\left\|v^{n}(0, \cdot)\right\|_{X_{2}(\rho)}+M \sup _{\tau \in[0, t], x \in \mathbb{R}^{N}}\left|a_{n}(\tau, x)\right| \int_{0}^{t} e^{\omega(t-\tau)}\left\|v^{n}(\tau, \cdot)\right\|_{X_{2}(\rho)} d \tau \\
& +\frac{M}{\omega} \sup _{\tau \in[0, t]}\left\|b_{n}(\tau, \cdot)\right\|_{X_{2}(\rho)} e^{\omega t} .
\end{aligned}
$$

By Gronwall's inequality,

$\left\|v^{n}(t, \cdot)\right\|_{X_{2}(\rho)} \leq e^{\left(\omega+M \sup _{\tau \in[0, t], x \in \mathbb{R}^{N}}\left|a_{n}(\tau, x)\right|\right) t}\left(M\left\|v^{n}(0, \cdot)\right\|_{X_{2}(\rho)}+\frac{M}{\omega} \sup _{\tau \in[0, t]}\left\|b_{n}(\tau, \cdot)\right\|_{X_{2}(\rho)}\right)$.

Note that $\left\|v^{n}(0, \cdot)\right\|_{X_{2}(\rho)} \rightarrow 0$ and $\sup _{\tau \in[0, t]}\left\|b_{n}(\tau, \cdot)\right\|_{X_{2}(\rho)} \rightarrow 0$ as $n \rightarrow \infty$. It then follows that

$$
\left\|v^{n}(t, \cdot)\right\|_{X_{2}(\rho)} \rightarrow 0 \quad \text { as } \quad n \rightarrow \infty
$$

and then

$$
u_{2}\left(t, x ; u_{0 n}, f_{2}\left(\cdot+z_{n}, \cdot\right)\right) \rightarrow u_{2}\left(t, x ; u_{0}, f_{2}\left(\cdot+z^{*}, \cdot\right)\right) \quad \text { as } \quad n \rightarrow \infty
$$

uniformly in $x$ on bounded sets. 
3.2. Principal eigenvalues of spatially periodic dispersal operators. In this subsection, we present some principal eigenvalue theories for spatially periodic dispersal operators with random, nonlocal, and discrete dispersals.

Let $p=\left(p_{1}, p_{2}, \ldots, p_{N}\right)$ with $p_{i}>0$ for $i=1,2, \cdots, N$ and $X_{i, p}$ be as in (2.1)(2.3). When $X_{3, p}$ is considered, it is assumed that $p_{i} \in \mathbb{N}$. We will denote $\mathcal{I}$ as an identity map on the Banach space under consideration. For given $\xi \in S^{N-1}, \mu \in \mathbb{R}$, $a_{i} \in X_{i, p}(i=1,2,3)$, consider the following eigenvalue problems,

$$
\left\{\begin{array}{l}
\Delta u(x)-2 \mu \xi \cdot \nabla u(x)+\left(a_{1}(x)+\mu^{2}\right) u(x)=\lambda u(x), \quad x \in \mathbb{R}^{N} \\
u\left(x+p_{i} \mathbf{e}_{\mathbf{i}}\right)=u(x), \quad x \in \mathbb{R}^{N}
\end{array}\right.
$$

$$
\left\{\begin{array}{l}
\int_{\mathbb{R}^{N}} e^{-\mu(y-x) \cdot \xi} \kappa(y-x) u(y) d y-u(x)+a_{2}(x) u(x)=\lambda u(x), \quad x \in \mathbb{R}^{N} \\
u\left(x+p_{i} \mathbf{e}_{\mathbf{i}}\right)=u(x), \quad x \in \mathbb{R}^{N}
\end{array}\right.
$$

and

$$
\left\{\begin{array}{l}
\sum_{k \in K} a_{k}\left(e^{-\mu k \cdot \xi} u(j+k)-u(j)\right)+a_{3}(j) u(j)=\lambda u(j), \quad j \in \mathbb{Z}^{N} \\
u\left(j+p_{i} \mathbf{e}_{\mathbf{i}}\right)=u(j), \quad j \in \mathbb{Z}^{N}
\end{array}\right.
$$

Observe that when $\mu=0,(3.4),(3.5)$, and (3.6) are independent of $\xi$. Observe also that if $u(t, x)=e^{-\mu\left(x \cdot \xi-\frac{\lambda}{\mu} t\right)} \phi(x)$ is a solution of

$$
u_{t}(t, x)=\Delta u(t, x)+a_{1}(x) u(t, x), \quad x \in \mathbb{R}^{N}
$$

with $\phi(\cdot) \in X_{1, p} \backslash\{0\}$, or a solution of

$$
u_{t}(t, x)=\int_{\mathbb{R}^{N}} k(y-x) u(t, y) d y-u(t, x)+a_{2}(x) u(t, x), \quad x \in \mathbb{R}^{N}
$$

with $\phi(\cdot) \in X_{2, p} \backslash\{0\}$, or a solution of

$$
u_{t}(t, j)=\sum_{k \in K} a_{k}(u(t, k+j)-u(t, j))+a_{3}(j) u(t, j), \quad j \in \mathbb{Z}^{N}
$$

with $\phi(\cdot) \in X_{3, p} \backslash\{0\}$, then $\lambda$ is an eigenvalue of (3.4) or (3.5) or (3.6) with $\phi(\cdot)$ being a corresponding eigenfunction. If $a_{1}(x)=f_{1}(x, 0)$ (resp. $a_{2}(x)=f_{2}(x, 0)$, $a_{3}(j)=f_{3}(j, 0)$ ), then $(3.7)$ (resp. (3.8), (3.9)) is the linearized equation of $(1.1)$ (resp. (1.2), (1.3)) at $u=0$.

Define $\mathcal{O}_{i, \mu, \xi}: \mathcal{D}\left(\mathcal{O}_{i, \mu, \xi}\right) \subset X_{i, p} \rightarrow X_{i, p}(i=1,2,3)$ by

$$
\left(\mathcal{O}_{1, \mu, \xi} u\right)(x)=\Delta u(x)-2 \mu \xi \cdot \nabla u(x)+\left(a_{1}(x)+\mu^{2}\right) u(x) \quad \forall u \in \mathcal{D}\left(\mathcal{O}_{1, \mu, \xi}\right) \subset X_{1, p},
$$

$\left(\mathcal{O}_{2, \mu, \xi} u\right)(x)=\int_{\mathbb{R}^{N}} e^{-\mu(y-x) \cdot \xi} \kappa(y-x) u(y) d y-u(x)+a_{2}(x) u(x) \quad \forall u \in \mathcal{D}\left(\mathcal{O}_{2, \mu, \xi}\right)=X_{2, p}$

and

$$
\left(\mathcal{O}_{3, \mu, \xi} u\right)(j)=\sum_{k \in K} a_{k}\left(e^{-\mu k \cdot \xi} u(j+k)-u(j)\right)+a_{3}(j) u(j) \quad \forall u \in \mathcal{D}\left(\mathcal{O}_{3, \mu, \xi}\right)=X_{3, p} .
$$


Let $\sigma\left(\mathcal{O}_{i, \mu, \xi}\right)$ be the spectrum of $\mathcal{O}_{i, \mu, \xi}(i=1,2,3)$.

Definition 3.1. Let $1 \leq i \leq 3, \mu \in \mathbb{R}$, and $\xi \in S^{N-1}$ be given. A real number $\lambda_{i}\left(\mu, \xi, a_{i}\right) \in \mathbb{R}$ is called the principal eigenvalue of $\mathcal{O}_{i, \mu, \xi}$ if it is an isolated algebraic simple eigenvalue of $\mathcal{O}_{i, \mu, \xi}$ with a positive eigenfunction and for any $\lambda \in$ $\sigma\left(\mathcal{O}_{i, \mu, \xi}\right) \backslash\left\{\lambda_{i}\left(\mu, \xi, a_{i}\right)\right\}, \operatorname{Re} \lambda<\lambda_{i}\left(\mu, \xi, a_{i}\right)$.

For given $1 \leq i \leq 3, \mu \in \mathbb{R}$, and $\xi \in S^{N-1}$, let

$$
\lambda_{i}^{0}\left(\mu, \xi, a_{i}\right)=\sup \left\{\operatorname{Re} \mu \mid \mu \in \sigma\left(\mathcal{O}_{i, \mu, \xi}\right)\right\} .
$$

Observe that for any $\mu \in \mathbb{R}$ and $\xi \in S^{N-1}, \mathcal{O}_{i, \mu, \xi}$ generates an analytic semigroup $\left\{T_{i}(t)\right\}_{t \geq 0}$ in $X_{i, p}$ and moreover, $T_{i}(t)$ is strongly positive (that is, $T_{i}(t) u_{0} \geq 0$ for any $t \geq 0$ and $u_{0} \in X_{i, p}^{+}$and $T_{i}(t) u_{0} \gg 0$ for any $t>0$ and $\left.u_{0} \in X_{i, p}^{+} \backslash\{0\}\right)$. Then by [53, Proposition 4.1.1], $r\left(T_{i}(t)\right) \in \sigma\left(T_{i}(t)\right)$ for any $t>0$, where $r\left(T_{i}(t)\right)$ is the spectral radius of $T_{i}(t)$. Hence by the spectral mapping theorem (see $[15$, Theorem $2.7]), \lambda_{i}^{0}\left(\mu, \xi, a_{i}\right) \in \sigma\left(\mathcal{O}_{i, \mu, \xi}\right)$ for $i=1,2,3$. Observe also that $\lambda_{i}^{0}\left(0, \xi, a_{i}\right)(i=1,2,3)$ are independent of $\xi \in S^{N-1}$. We may then put

$$
\lambda_{i}^{0}\left(a_{i}\right)=\lambda_{i}^{0}\left(0, \xi, a_{i}\right), \quad i=1,2,3 .
$$

It is well known that the principal eigenvalue $\lambda_{1}\left(\mu, \xi, a_{1}\right)$ and $\lambda_{3}\left(\mu, \xi, a_{3}\right)$ of $\mathcal{O}_{1, \mu, \xi}$ and $\mathcal{O}_{3, \mu, \xi}$ exist for all $\mu \in \mathbb{R}$ and $\xi \in S^{N-1}$ and

$$
\lambda_{i}\left(\mu, \xi, a_{i}\right)=\lambda_{i}^{0}\left(\mu, \xi, a_{i}\right), \quad i=1,3 .
$$

The principal eigenvalue of $\mathcal{O}_{2, \mu, \xi}$ may not exist (see [19] and [64] for examples). If the principal eigenvalue $\lambda_{2}\left(\mu, \xi, a_{2}\right)$ exists, then

$$
\lambda_{2}\left(\mu, \xi, a_{2}\right)=\lambda_{2}^{0}\left(\mu, \xi, a_{2}\right)
$$

Regarding the existence of principal eigenvalue of $\mathcal{O}_{2, \mu, \xi}$, the following proposition is proved in [64], [65].

Proposition 3.5 (Existence of principal eigenvalue).

(1) If $a_{2} \in C^{N}\left(\mathbb{R}^{N}, \mathbb{R}\right) \cap X_{2, p}$ and the partial derivatives of $a_{2}(x)$ up to order $N-1$ are zero at some $x_{0}$ satisfying that $a_{2}\left(x_{0}\right)=\max _{x \in \mathbb{R}^{N}} a_{2}(x)$, then the principal eigenvalue $\lambda_{2}\left(\mu, \xi, a_{2}\right)$ of $\mathcal{O}_{2, \mu, \xi}$ exists for all $\mu \in \mathbb{R}$ and $\xi \in S^{N-1}$.

(2) If $a_{2}(x)$ satisfies that $\max _{x \in \mathbb{R}^{N}} a_{2}(x)-\min _{x \in \mathbb{R}^{N}} a_{2}(x)<$ $\inf _{\xi \in S^{N-1}} \int_{z \cdot \xi \leq 0} k(z) d z$, then the principal eigenvalue $\lambda_{2}\left(\mu, \xi, a_{2}\right)$ of $\mathcal{O}_{2, \mu, \xi}$ exists for all $\mu \in \mathbb{R}$ and $\xi \in S^{N-1}$.

Proof. (1) It follows from [64, Theorem B].

(2) It follows from $\left[65\right.$, Theorem $\left.\mathrm{B}^{\prime}\right]$.

Let $\hat{a}_{i}$ be the average of $a_{i}(\cdot)(i=1,2,3)$, that is,

$$
\left\{\begin{array}{l}
\hat{a}_{i}=\frac{1}{\left|D_{i}\right|} \int_{D_{i}} a_{i}(x) d x \quad \text { for } \quad i=1,2 \\
\hat{a}_{3}=\frac{1}{\# D_{3}} \sum_{j \in D_{3}} a_{3}(j)
\end{array}\right.
$$

where

$$
D_{i}=\left[0, p_{1}\right] \times\left[0, p_{2}\right] \times \cdots \times\left[0, p_{N}\right] \cap \mathcal{H}_{i}, i=1,2,3
$$


and

$$
\left\{\begin{array}{l}
\left|D_{i}\right|=p_{1} \times p_{2} \times \cdots \times p_{N} \text { for } i=1,2 \\
\# D_{3}=\text { the cardinality of } D_{3} .
\end{array}\right.
$$

By Proposition $3.5(2), \lambda_{2}\left(\mu, \xi, \hat{a}_{2}\right)$ exists for all $\mu \in \mathbb{R}$ and $\xi \in S^{N-1}$. The following proposition shows a relation between $\lambda_{i}^{0}\left(\mu, \xi, a_{i}\right)$ and $\lambda_{i}^{0}\left(\mu, \xi, \hat{a}_{i}\right)$.

Proposition 3.6 (Influence of spatial variation). For given $1 \leq i \leq 3, \mu \in \mathbb{R}$, and $\xi \in S^{N-1}$, there holds

$$
\lambda_{i}^{0}\left(\mu, \xi, a_{i}\right) \geq \lambda_{i}^{0}\left(\mu, \xi, \hat{a}_{i}\right) .
$$

Proof. The case $i=1$ is well known. The cases $i=2$ and 3 follow from [33, Theorem 2.1].

We remark that $\lambda_{i}\left(\mu, \xi, \hat{a}_{i}\right)\left(=\lambda_{i}^{0}\left(\mu, \xi, \hat{a}_{i}\right)\right)(i=1,2,3)$ have the following explicit expressions,

$$
\left\{\begin{array}{l}
\lambda_{1}\left(\mu, \xi, \hat{a}_{1}\right)=\hat{a}_{1}+\mu^{2} \\
\lambda_{2}\left(\mu, \xi, \hat{a}_{2}\right)=\int_{\mathbb{R}^{N}} e^{-\mu z \cdot \xi} \kappa(z) d z-1+\hat{a}_{2} \\
\lambda_{3}\left(\mu, \xi, \hat{a}_{3}\right)=\sum_{k \in K} a_{k}\left(e^{-\mu k \cdot \xi}-1\right)+\hat{a}_{3} .
\end{array}\right.
$$

3.3. KPP equations in spatially periodic media. In this subsection, we recall some spatial spreading dynamics of KPP equations in spatially periodic media.

Consider

$$
\begin{gathered}
u_{t}(t, x)=\Delta u(t, x)+u(t, x) g_{1}(x, u(t, x)), \quad x \in \mathbb{R}^{N}, \\
u_{t}(t, x)=\int_{\mathbb{R}^{N}} \kappa(y-x) u(t, y) d y-u(t, x)+u(t, x) g_{2}(x, u(t, x)), \quad x \in \mathbb{R}^{N},
\end{gathered}
$$

and

$$
u_{t}(t, j)=\sum_{k \in K} a_{k}(u(t, j+k)-u(t, j))+u(t, j) g_{3}(j, u(t, j)), \quad j \in \mathbb{Z}^{N},
$$

where $g_{i}(\cdot, \cdot)(i=1,2,3)$ are periodic in the first variable and monostable in the second variable. More precisely, we assume

(P1) $1 \leq i \leq 3$ and $g_{i}: \mathcal{H}_{i} \times \mathbb{R} \rightarrow \mathbb{R}$ is a $C^{2}$ function, $g_{i}\left(x+p_{l} \mathbf{e}_{l}, u\right)=g_{i}(x, u)$, where $p_{l}>0$ and $p_{l} \in \mathbb{N}$ in the case $i=3(l=1,2, \cdots, N)$, and $g_{i}(x, u)<0$ for all $(x, u) \in \mathcal{H}_{i} \times \mathbb{R}^{+}$with $u \geq \alpha_{0}$ for some $\alpha_{0}>0$ and $\partial_{u} g_{i}(x, u)<0$ for all $(x, u) \in \mathcal{H}_{i} \times \mathbb{R}^{+}$.

(P2) $\lambda_{i}^{0}\left(g_{i}(\cdot, 0)\right)>0$, where $i=1,2,3$.

Assume (P1). Similarly, by general semigroup theory, for any $u_{0} \in X_{1}$ (resp. $\left.u_{0} \in X_{2}, u_{0} \in X_{3}\right)$, (3.18) (resp. (3.19), (3.20)) has a unique (local) solution $u_{1}\left(t, \cdot ; u_{0}, g_{1}(\cdot, \cdot)\right)\left(\in X_{1}\right)\left(\right.$ resp. $\left.u_{2}\left(t, \cdot ; u_{0}, g_{2}(\cdot, \cdot)\right)\left(\in X_{2}\right), u_{3}\left(t, \cdot ; u_{0}, g_{3}(\cdot, \cdot)\right)\left(\in X_{3}\right)\right)$ with initial data $u_{0}(\cdot)$. Moreover, if $u_{0} \in X_{i, p}$, then $u_{i}\left(t, \cdot ; u_{0}, g_{i}(\cdot, \cdot)\right) \in X_{i, p}$ for any $t>0$ at which $u_{i}\left(t, \cdot ; u_{0}, g_{i}(\cdot, \cdot)\right)$ exists $(i=1,2,3)$. By Proposition 3.1, if $u_{0} \in X_{i}^{+}$, then $u_{i}\left(t, \cdot ; u_{0}, g_{i}(\cdot, \cdot)\right)$ exists and $u_{i}\left(t, \cdot ; u_{0}, g_{i}(\cdot, \cdot)\right) \in X_{i}^{+}$for all $t>0(i=1,2,3)$. 
Proposition 3.7 (Spatially periodic positive stationary solution). Assume (P1) and (P2). Then (3.18) (resp. (3.19), (3.20)) has a unique spatially periodic stationary solution $u_{1}^{*}\left(\cdot ; g_{1}(\cdot, \cdot)\right) \in X_{1, p}^{++}\left(\right.$resp. $\left.u_{2}^{*}\left(\cdot ; g_{2}(\cdot, \cdot)\right) \in X_{2, p}^{++}, u_{3}^{*}\left(\cdot ; g_{3}(\cdot, \cdot)\right) \in X_{3, p}^{++}\right)$which is globally asymptotically stable with respect to perturbations in $X_{1, p}^{+} \backslash\{0\}\left(\right.$ resp. $X_{2, p}^{+} \backslash$ $\left.\{0\}, X_{3, p}^{+} \backslash\{0\}\right)$.

Proof. The cases that $i=1$ and 3 follow from [75, Theorem 2.3]. The case that $i=2$ follows from [65, Theorem $\mathrm{C}]$.

Proposition 3.8 (Spreading speeds). Assume (P1) and (P2). Then for any $\xi \in S^{N-1}$, (3.18) (resp. (3.19), (3.20)) has a positive spreading speed $c_{1}^{*}\left(\xi ; g_{1}(\cdot, \cdot)\right)$ $\left(\right.$ resp. $\left.c_{2}^{*}\left(\xi ; g_{2}(\cdot, \cdot)\right), c_{3}^{*}\left(\xi ; g_{3}(\cdot, \cdot)\right)\right)$ in the direction of $\xi$. Moreover,

$$
c_{i}^{*}\left(\xi ; g_{i}(\cdot, \cdot)\right)=\inf _{\mu>0} \frac{\lambda_{i}^{0}\left(\mu, \xi, g_{i}(\cdot, 0)\right)}{\mu} \quad(i=1,2,3)
$$

and the following hold for $i=1,2,3$.

(1) For each $u_{0} \in X_{i}^{+}$satisfying that $u_{0}(x)=0$ for $x \in \mathcal{H}_{i}$ with $|x \cdot \xi| \gg 1$,

$$
\limsup _{|x \cdot \xi| \geq c t, t \rightarrow \infty} u_{i}\left(t, x ; u_{0}, g_{i}(\cdot, \cdot)\right)=0 \quad \forall c>\max \left\{c_{i}^{*}\left(\xi ; g_{i}(\cdot, \cdot)\right), c_{i}^{*}\left(-\xi ; g_{i}(\cdot, \cdot)\right)\right\} .
$$

(2) For each $\sigma>0, r>0$, and $u_{0} \in X_{i}^{+}$satisfying that $u_{0}(x) \geq \sigma$ for $x \in \mathcal{H}_{i}$ with $|x \cdot \xi| \leq r$

$$
\limsup _{|x \cdot \xi| \leq c t, t \rightarrow \infty}\left|u_{i}\left(t, x ; u_{0}, g_{i}(\cdot, \cdot)\right)-u_{i}^{*}\left(x ; g_{i}(\cdot, \cdot)\right)\right|=0
$$

for all $0<c<\min \left\{c_{i}^{*}\left(\xi ; g_{i}(\cdot, \cdot)\right), c_{i}^{*}\left(-\xi ; g_{i}(\cdot, \cdot)\right)\right\}$.

(3) For each $u_{0} \in X_{i}^{+}$satisfying that $u_{0}(x)=0$ for $x \in \mathcal{H}_{i}$ with $\|x\| \gg 1$,

$$
\limsup _{\|x\| \geq c t, t \rightarrow \infty} u_{i}\left(t, x ; u_{0}, g_{i}(\cdot, \cdot)\right)=0 \quad \forall c>\sup _{\xi \in S^{N-1}} c_{i}^{*}\left(\xi ; g_{i}(\cdot, \cdot)\right) .
$$

(4) For each $\sigma>0, r>0$, and $u_{0} \in X_{i}^{+}$satisfying that $u_{0}(x) \geq \sigma$ for $x \in \mathcal{H}_{i}$ with $\|x\| \leq r$,

$$
\limsup _{\|x\| \leq c t, t \rightarrow \infty}\left|u_{i}\left(t, x ; u_{0}, g_{i}(\cdot, \cdot)\right)-u_{i}^{*}\left(x ; g_{i}(\cdot, \cdot)\right)\right|=0 \quad \forall 0<c<\inf _{\xi \in S^{N-1}} c_{i}^{*}\left(\xi ; g_{i}(\cdot, \cdot)\right) .
$$

Proof. The cases $i=1$ and $i=3$ follow from [45, Theorems 3.1-3.4 and Corollary 3.1] (see also [72, Theorems 2.1-2.3]) and the case $i=2$ follows from [65, Theorems $\mathrm{D}$ and $\mathrm{E}]$.

Let $\hat{g}_{1}(u)$ (resp. $\left.\hat{g}_{2}(u), \hat{g}_{3}(u)\right)$ be the spatial average of $g_{1}(x, u)$ (resp. $g_{2}(x, u)$, $\left.g_{3}(x, u)\right)$, respectively, that is,

$$
\left\{\begin{array}{l}
\hat{g}_{i}(u)=\frac{1}{\left|D_{i}\right|} \int_{D_{i}} g_{i}(x, u) d x \quad \text { for } \quad i=1,2 \\
\hat{g}_{3}(u)=\frac{1}{\# D_{3}} \sum_{j \in D_{3}} g_{3}(j, u),
\end{array}\right.
$$

where $D_{i}(i=1,2,3),\left|D_{i}\right|(i=1,2)$ and $\# D_{3}$ are as in (3.15) and (3.16).

Assume

(P3) $\hat{g}_{i}(0)>0(i=1,2,3)$. 
Observe that $\lambda_{i}\left(\hat{g}_{i}(0)\right)=\hat{g}_{i}(0)$. Then by Proposition 3.6, (P3) implies (P2). Assume (P3). By Proposition 3.8, for any $\xi \in S^{N-1}$, (3.18) (resp. (3.19), (3.20)) with $g_{1}(x, u)$ (resp. $\left.g_{2}(x, u), g_{3}(j, u)\right)$ being replaced by $\hat{g}_{1}(u)$ (resp. $\hat{g}_{2}(u), \hat{g}_{3}(u)$ ) has a spreading speed $c_{1}^{*}\left(\xi ; \hat{g}_{1}(\cdot)\right)\left(\operatorname{resp} . c_{2}^{*}\left(\xi ; \hat{g}_{2}(\cdot)\right), c_{3}^{*}\left(\xi ; \hat{g}_{3}(\cdot)\right)\right)$ in the direction of $\xi \in S^{N-1}$.

Proposition 3.9 (Influence of spatial variation). Assume (P1) and (P3). Then for any $\xi \in S^{N-1}$,

$$
c_{i}^{*}\left(\xi ; g_{i}(\cdot, \cdot)\right) \geq c_{i}^{*}\left(\xi ; \hat{g}_{i}(\cdot)\right), \quad i=1,2,3 .
$$

Proof. Let $a_{i}(\cdot)=g_{i}(\cdot, 0)$. By Proposition 3.8,

$$
c_{i}^{*}\left(\xi ; g_{i}(\cdot, \cdot)\right)=\inf _{\mu>0} \frac{\lambda_{i}^{0}\left(\mu, \xi, a_{i}\right)}{\mu} \quad \text { and } \quad c_{i}^{*}\left(\xi ; \hat{g}_{i}(\cdot)\right)=\inf _{\mu>0} \frac{\lambda_{i}^{0}\left(\mu, \xi, \hat{a}_{i}\right)}{\mu}
$$

for $i=1,2,3$. By Proposition 3.6,

$$
\lambda_{i}^{0}\left(\mu, \xi, a_{i}\right) \geq \lambda_{i}^{0}\left(\mu, \xi, \hat{a}_{i}\right) \quad i=1,2,3 .
$$

The proposition then follows. $\square$

4. Positive stationary solutions and the proof of Theorem 2.1. In this section, we investigate the existence of positive stationary solutions of (1.1), (1.2), and (1.3), and prove Theorem 2.1.

Throughout this section, we assume (H1) and (H2). We first prove some lemmas.

Lemma 4.1. For any $1 \leq i \leq 3$ and $\epsilon>0$, there are $p=\left(p_{1}, p_{2}, \cdots, p_{N}\right) \in \mathbb{N}^{N}$ and $h_{i} \in X_{i, p} \cap C^{N}\left(\mathcal{H}_{i}, \mathbb{R}\right)$ such that

$$
\begin{gathered}
f_{i}(x, 0) \geq h_{i}(x) \quad \text { for } \quad x \in \mathcal{H}_{i}, \\
\hat{h}_{i} \geq f_{i}^{0}(0)-\epsilon \quad\left(\text { hence } \quad \lambda_{i}^{0}\left(h_{i}(\cdot)\right) \geq f_{i}^{0}(0)-\epsilon\right),
\end{gathered}
$$

and for the cases that $i=1$ and 2 , the partial derivatives of $h_{i}(x)$ up to order $N-1$ are zero at some $x_{0} \in \mathcal{H}_{i}$ with $h_{i}\left(x_{0}\right)=\max _{x \in \mathcal{H}_{i}} h_{i}(x)$, where $\hat{h}_{i}$ is the average of $h_{i}(\cdot)$ (see (3.14) for the definition).

Proof. Fix $1 \leq i \leq 3$. By (H2), there is $L_{0}>0$ such that $f_{i}(x, 0)=f_{i}^{0}(0)$ for $x \in \mathcal{H}_{i}$ with $\|x\| \geq L_{0}$. Let $M_{0}=\inf _{x \in \mathcal{H}_{i}, 1 \leq i \leq 3} f_{i}(x, 0)$. Let $h_{0}: \mathbb{R} \rightarrow[0,1]$ be a smooth function such that $h_{0}(s)=1$ for $|s| \leq 1$ and $h_{0}(s)=0$ for $|s| \geq 2$. For any $p=\left(p_{1}, p_{2}, \cdots, p_{N}\right) \in \mathbb{N}^{N}$ with $p_{j}>4 L_{0}$, let $h_{i} \in X_{i, p} \cap C^{N}\left(\mathcal{H}_{i}, \mathbb{R}\right)(i=1,2,3)$ be such that

$$
\begin{gathered}
h_{i}(x)=f_{i}^{0}(0)-h_{0}\left(\frac{\|x\|^{2}}{L_{0}^{2}}\right)\left(f_{i}^{0}(0)-M_{0}\right) \\
\text { for } x \in\left(\left[-\frac{p_{1}}{2}, \frac{p_{1}}{2}\right] \times\left[-\frac{p_{2}}{2}, \frac{p_{2}}{2}\right] \times \cdots \times\left[-\frac{p_{N}}{2}, \frac{p_{N}}{2}\right]\right) \cap \mathcal{H}_{i} .
\end{gathered}
$$

Then

$$
f_{i}(x, 0) \geq h_{i}(x) \quad \forall x \in \mathcal{H}_{i}, 1 \leq i \leq 3 .
$$


It is clear that for $i=1$ or 2 , the partial derivatives of $h_{i}(x)$ up to order $N-1$ are zero at some $x_{0} \in \mathcal{H}_{i}$ with $h_{i}\left(x_{0}\right)=\max _{x \in \mathcal{H}_{i}} h_{i}(x)\left(=f_{i}^{0}(0)\right)$. For given $\epsilon>0$, choosing $p_{j} \gg 1$, we have

$$
\hat{h}_{i}>f_{i}^{0}(0)-\epsilon .
$$

By Proposition 3.6, $\lambda_{i}^{0}\left(h_{i}(\cdot)\right) \geq \lambda_{i}^{0}\left(\hat{h}_{i}\right)=\hat{h}_{i}$ and hence

$$
\lambda_{i}^{0}\left(h_{i}(\cdot)\right) \geq f_{i}^{0}(0)-\epsilon .
$$

The lemma is thus proved.

Lemma 4.2. Suppose that $\tilde{u}_{2}^{*}: \mathbb{R}^{N} \rightarrow\left[\sigma_{0}, M_{0}\right]$ is Lebesgue measurable, where $\sigma_{0}$ and $M_{0}$ are two positive constants. If

$$
\int_{\mathbb{R}^{N}} \kappa(y-x) \tilde{u}_{2}^{*}(y) d y-\tilde{u}_{2}^{*}(x)+\tilde{u}_{2}^{*}(x) \tilde{f}_{2}\left(x, \tilde{u}_{2}^{*}(x)\right)=0 \quad \forall x \in \mathbb{R}^{N},
$$

where $\tilde{f}_{2}(x, u)=f_{2}(x, u)$ or $f_{2}^{0}(u)$ for all $x \in \mathbb{R}^{N}$ and $u \in \mathbb{R}$, then $\tilde{u}_{2}^{*}(\cdot) \in X_{2}^{++}$.

Proof. We prove the case that $\tilde{f}_{2}(x, u)=f_{2}(x, u)$. The case that $\tilde{f}_{2}(x, u)=f_{2}^{0}(u)$ can be proved similarly.

Let $h^{*}(x)=\int_{\mathbb{R}^{N}} \kappa(y-x) \tilde{u}_{2}^{*}(y) d y$ for $x \in \mathbb{R}^{N}$. Then $h^{*}(\cdot)$ is $C^{1}$ and has bounded first order partial derivatives. Let

$$
F(x, \alpha)=h^{*}(x)-\alpha+\alpha f_{2}(x, \alpha) \quad \forall x \in \mathbb{R}^{N}, \alpha \in \mathbb{R} .
$$

Then $F: \mathbb{R}^{N} \times \mathbb{R} \rightarrow \mathbb{R}$ is $C^{1}$ and $F\left(x, \tilde{u}_{2}^{*}(x)\right)=0$ for each $x \in \mathbb{R}^{N}$. If $\alpha^{*}>0$ is such that $F\left(x, \alpha^{*}\right)=0$, then

$$
-1+f_{2}\left(x, \alpha^{*}\right)=-\frac{h^{*}(x)}{\alpha^{*}}<0
$$

and hence

$$
\partial_{\alpha} F\left(x, \alpha^{*}\right)=-1+f_{2}\left(x, \alpha^{*}\right)+\alpha^{*} \partial_{u} f_{2}\left(x, \alpha^{*}\right)<0 .
$$

By Implicit Function Theorem, $\tilde{u}_{2}^{*}(x)$ is $C^{1}$ in $x$. Moreover,

$$
\frac{\partial \tilde{u}_{2}^{*}(x)}{\partial x_{j}}=\frac{\frac{\partial h^{*}(x)}{\partial x_{j}}}{-1+f_{2}\left(x, \tilde{u}_{2}^{*}(x)\right)+\partial_{u} f_{2}\left(x, \tilde{u}_{2}^{*}(x)\right) \tilde{u}_{2}^{*}(x)} \quad \forall x \in \mathbb{R}^{N}, 1 \leq j \leq N .
$$

Therefore, $\tilde{u}_{2}^{*}$ has bounded first order partial derivatives. It then follows that $\tilde{u}_{2}^{*}(x)$ is uniformly continuous in $x \in \mathbb{R}^{N}$ and then $\tilde{u}_{2}^{*} \in X_{2}^{++}$. $\square$

LEMma 4.3. Suppose that $u_{i}^{*}(\cdot) \in X_{i}^{++}$and $u=u_{i}^{*}(\cdot)$ is a stationary solution of (1.i) $(1 \leq i \leq 3)$. Then

$$
u_{i}^{*}(x) \rightarrow u_{i}^{0} \quad \text { as } \quad\|x\| \rightarrow \infty .
$$

Proof. We first prove that

$$
u_{1}^{*}(x) \rightarrow u_{1}^{0} \quad \text { as } \quad\|x\| \rightarrow \infty .
$$


Assume that $u_{1}^{*}(x) \not \rightarrow u_{1}^{0}$ as $\|x\| \rightarrow \infty$. Then there are $\epsilon_{0}>0$ and $x_{n} \in \mathbb{R}^{N}$ such that $\left\|x_{n}\right\| \rightarrow \infty$ and

$$
\left|u_{1}^{*}\left(x_{n}\right)-u_{1}^{0}\right| \geq \epsilon_{0} \quad \text { for } \quad n=1,2, \cdots .
$$

By the uniform continuity of $u_{1}^{*}(x)$ in $x \in \mathbb{R}^{N}$, without loss of generality, we may assume that there is a continuous function $\tilde{u}_{1}^{*}: \mathbb{R}^{N} \rightarrow\left[\sigma_{0}, M_{0}\right]$ for some $\sigma_{0}, M_{0}>0$ such that

$$
u_{1}\left(x+x_{n}\right) \rightarrow \tilde{u}_{1}^{*}(x)
$$

as $n \rightarrow \infty$ uniformly in $x$ on bounded sets. Moreover, by a priori estimates for parabolic equations, $\tilde{u}_{1}^{*}$ is $C^{2+\alpha}$ for some $\alpha>0$ and we may also assume that

$$
\Delta u_{1}\left(x+x_{n}\right) \rightarrow \Delta \tilde{u}_{1}^{*}(x)
$$

as $n \rightarrow \infty$ uniformly in $x$ on bounded sets. This together with $f_{1}\left(x+x_{n}, u\right) \rightarrow f_{1}^{0}(u)$ as $n \rightarrow \infty$ uniformly in $x$ on bounded sets and in $u \in \mathbb{R}$ implies that

$$
\Delta \tilde{u}_{1}^{*}+\tilde{u}_{1}^{*} f_{1}^{0}\left(\tilde{u}_{1}^{*}\right)=0, \quad x \in \mathbb{R}^{N} .
$$

By Proposition 3.7, we must have $\tilde{u}_{1}^{*}(x) \equiv u_{1}^{*}\left(x ; f_{1}^{0}(\cdot)\right) \equiv u_{1}^{0}$ and hence $u_{1}^{*}\left(x_{n}\right) \rightarrow u_{1}^{0}$ as $n \rightarrow \infty$. This is a contradiction. Therefore $u_{1}^{*}(x) \rightarrow u_{1}^{0}$ as $\|x\| \rightarrow \infty$.

Next, we prove that

$$
u_{2}^{*}(x) \rightarrow u_{2}^{0} \quad \text { as } \quad\|x\| \rightarrow \infty .
$$

Similarly, assume that $u_{2}^{*}(x) \nrightarrow \rightarrow u_{2}^{0}$ as $\|x\| \rightarrow \infty$. Then there are $\epsilon_{0}>0$ and $x_{n} \in \mathbb{R}^{N}$ such that $\left\|x_{n}\right\| \rightarrow \infty$ and

$$
\left|u_{2}^{*}\left(x_{n}\right)-u_{2}^{0}\right| \geq \epsilon_{0} \quad \text { for } \quad n=1,2, \cdots .
$$

By the uniform continuity of $u_{2}^{*}(x)$ in $x \in \mathbb{R}^{N}$, without loss of generality, we may assume that there is a continuous function $\tilde{u}_{2}^{*}: \mathbb{R}^{N} \rightarrow\left[\sigma_{0}, M_{0}\right]$ for some $\sigma_{0}, M_{0}>0$ such that

$$
u_{2}\left(x+x_{n}\right) \rightarrow \tilde{u}_{2}^{*}(x)
$$

as $n \rightarrow \infty$ uniformly in $x$ on bounded sets. By the Lebesgue Dominated Convergence Theorem, we have

$$
\int_{\mathbb{R}^{N}} \kappa(y-x) \tilde{u}_{2}^{*}(y) d y-\tilde{u}_{2}^{*}(x)+\tilde{u}_{2}^{*}(x) f_{2}^{0}\left(\tilde{u}_{2}^{*}(x)\right)=0 \quad \forall x \in \mathbb{R}^{N} .
$$

By Lemma 4.2, $\tilde{u}_{2}^{*} \in X_{2}^{++}$. By Proposition 3.7 again, we have $\tilde{u}_{2}^{*}(x) \equiv u_{2}^{0}$ and then $u_{2}^{*}\left(x_{n}\right) \rightarrow u_{2}^{0}$ as $n \rightarrow \infty$. This is a contradiction. Therefore $u_{2}^{*}(x) \rightarrow u_{2}^{0}$ as $\|x\| \rightarrow \infty$.

Finally, it can be proved by the similar arguments as in the case $i=2$ that

$$
u_{3}^{*}(j) \rightarrow u_{3}^{0} \quad \text { as } \quad\|j\| \rightarrow \infty .
$$

Lemma 4.4. There is $u_{i}^{-} \in X_{i}^{++}$such that for any $\delta>0$ sufficiently small, $u_{i}\left(t, x ; \delta u_{i}^{-}\right)$is increasing in $t>0$ and $u_{i}^{-, *, \delta} \in X_{i}^{++}$, where $u_{i}^{-, *, \delta}(x)=$ 
$\lim _{t \rightarrow \infty} u_{i}\left(t, x ; \delta u_{i}^{-}\right)$, and hence $u=u_{i}^{-, *, \delta}(\cdot)$ is a stationary solution of (1.i) in $X_{i}^{++}$ $(i=1,2,3)$.

Proof. Fix $1 \leq i \leq 3$. Let $M^{*}>0$ be such that $f_{i}\left(x, M^{*}\right)<0$. Let $\epsilon>0$ be such that

$$
f_{i}^{0}(0)-\epsilon>0
$$

By Lemma 4.1, there are $p \in \mathbb{N}^{N}$ and $h_{i}(\cdot) \in X_{i, p} \cap C^{N}\left(\mathcal{H}_{i}, \mathbb{R}\right)$ such that

$$
f_{i}(x, 0) \geq h_{i}(x), \text { and } \hat{h}_{i} \geq f_{i}^{0}(0)-\epsilon(>0) .
$$

Moreover, for $i=1$ or 2 , the partial derivatives of $h_{i}(x)$ up to order $N-1$ are zero at some $x_{0} \in \mathcal{H}_{i}$ with $h_{i}\left(x_{0}\right)=\max _{x \in \mathcal{H}_{i}} h_{i}(x)$. Let $u_{i}^{-}$be the positive principal eigenfunction of $\mathcal{O}_{i, 0,0}$ with $a_{i}(\cdot)=h_{i}(\cdot)$ and $\left\|u_{i}^{-}\right\|=1$ (the existence of $u_{i}^{-}$is well known in the case that $i=1$ or 3 and follows from Proposition 3.5 in the case that $i=2$ ). It is not difficult to verify that $u=\delta u_{i}^{-}$is a sub-solution of (1.i) for any $\delta>0$ sufficiently small. It then follows that for any $\delta>0$ sufficiently small,

$$
\delta u_{i}^{-}(\cdot) \leq u_{i}\left(t_{1}, \cdot ; \delta u_{i}^{-}\right) \leq u_{i}\left(t_{2}, \cdot ; \delta u_{i}^{-}\right) \quad \forall 0<t_{1}<t_{2} .
$$

This implies that there is a Lebesgue measurable function $u_{i}^{-, *, \delta}: \mathcal{H}_{i} \rightarrow\left[\sigma_{0}, M_{0}\right]$ for some $\sigma_{0}, M_{0}>0$ such that

$$
\lim _{t \rightarrow \infty} u_{i}\left(t, x ; \delta u_{i}^{-}\right)=u_{i}^{-, *, \delta}(x) \quad \forall x \in \mathcal{H}_{i} .
$$

Moreover, by regularity and a priori estimates for parabolic equations, $u_{1}^{-, *, \delta} \in X_{1}^{++}$. It is clear that $u_{3}^{-, *, \delta} \in X_{3}^{++}$. By Lemma $4.2, u_{2}^{-, *, \delta} \in X_{2}^{++}$. Therefore for $1 \leq i \leq 3$, $u_{i}^{-, *, \delta} \in X_{i}^{++}$and $u=u_{i}^{-, *, \delta}(\cdot)$ is a stationary solution of $(1 . \mathrm{i})$ in $X_{i}^{++}(i=1,2,3)$.

LEMMA 4.5. Let $M \gg 1$ be such that $f_{i}(x, M)<0$ for $x \in \mathcal{H}_{i}(i=1,2,3)$. Then $\lim _{t \rightarrow \infty} u_{i}\left(t, x ; u_{0}\right)$ exists for every $x \in \mathcal{H}_{i}$, where $u_{0}(x) \equiv M$. Moreover, $u_{i}^{+, *, M}(\cdot) \in$ $X_{i}^{++}$, where $u_{i}^{+, *, M}(x):=\lim _{t \rightarrow \infty} u_{i}\left(t, x ; u_{0}\right)$, and hence $u=u_{i}^{+, *, M}(\cdot)$ is a stationary solution of (1.i) in $X_{i}^{++}(i=1,2,3)$.

Proof. Fix $1 \leq i \leq 3$. For any $M>1$ with $f_{i}(x, M)<0$ for all $x \in \mathcal{H}_{i}, u=M$ is a super-solution of (1.i). Hence

$$
u_{i}\left(t_{2}, \cdot ; M\right) \leq u_{i}\left(t_{1}, \cdot ; M\right) \leq M \quad \forall 0 \leq t_{1}<t_{2} .
$$

It then follows that $\lim _{t \rightarrow \infty} u_{i}(t, x ; M)$ exists for all $x \in \mathbb{R}^{N}$. Let $u_{i}^{+, *, M}(x)=$ $\lim _{t \rightarrow \infty} u_{i}(t, x ; M)$. We have $u_{i}^{+, *, M}(x) \geq u_{i}^{-, *, \delta}(x)$ for $0<\delta \ll 1$. By the similar arguments as in Lemma 4.4, $u_{i}^{+, *, M} \in X_{i}^{++}$and $u=u_{i}^{+, *, M}(\cdot)$ is a stationary solution of (1.i) in $X_{i}^{++}(i=1,2,3)$.

Proof of Theorem 2.1. (1) Let $1 \leq i \leq 3$ be given. First, by Lemmas 4.4 and $4.5,(1 . \mathrm{i})$ has stationary solutions in $X_{i}^{++}$. We claim that stationary solution of (1.i) in $X_{i}^{++}$is unique. In fact, suppose that $u_{i}^{1, *}$ and $u_{i}^{2, *}$ are two stationary solutions of $(1 . \mathrm{i})$ in $X_{i}^{++}$. Assume that $u_{i}^{1, *} \neq u_{i}^{2, *}$. Then there is $\alpha^{*}>1$ such that $\rho_{i}\left(u_{i}^{1, *}, u_{i}^{2, *}\right)=\ln \alpha^{*}>0$. Note that

$$
\frac{1}{\alpha^{*}} u_{i}^{1, *} \leq u_{i}^{2, *} \leq \alpha^{*} u_{i}^{1, *} .
$$


By Lemma $4.3, \lim _{\|x\| \rightarrow \infty} u_{i}^{1, *}(x)=u_{i}^{0}$ and $\lim _{\|x\| \rightarrow \infty} u_{i}^{2, *}(x)=u_{i}^{0}$. This implies that there is $\epsilon>0$ such that

$$
\frac{1}{\alpha^{*}-\epsilon} u_{i}^{1, *}(x) \leq u_{i}^{2, *}(x) \leq\left(\alpha^{*}-\epsilon\right) u_{i}^{1, *}(x) \quad \text { for } \quad\|x\| \gg 1 .
$$

By Proposition 3.1 and the arguments in Proposition 3.3,

$$
\frac{1}{\alpha^{*}} u_{i}^{1, *}(x)<u_{i}^{2, *}(x)<\alpha^{*} u_{i}^{1, *}(x) \quad \forall x \in \mathbb{R}^{N} .
$$

It then follows that for $0<\epsilon \ll 1$,

$$
\frac{1}{\alpha^{*}-\epsilon} u_{i}^{1, *}(x) \leq u_{i}^{2, *}(x) \leq\left(\alpha^{*}-\epsilon\right) u_{i}^{1, *}(x) \quad \forall x \in \mathbb{R}^{N}
$$

and then $\rho_{i}\left(u_{i}^{1, *}, u_{i}^{2, *}\right) \leq \ln \left(\alpha^{*}-\epsilon\right)$, this is a contradiction. Therefore $u_{i}^{1, *}=u_{i}^{2, *}$ and (1.i) has a unique stationary solution $u_{i}^{*}$ in $X_{i}^{++}$.

(2) Fix $1 \leq i \leq 3$. For any $u_{0} \in X_{i}^{++}$, there is $\delta>0$ sufficiently small and $M>0$ sufficiently large such that $\delta u_{i}^{-} \leq u_{0} \leq M$ and $u=\delta u_{i}^{-}$is a sub-solution of (1.i) $\left(u_{i}^{-}\right.$ is as in Lemma 4.4) and $u=M$ is a super-solution of (1.i). Then

$$
\delta u_{i}^{-} \leq u_{i}\left(t, \cdot ; \delta u_{i}^{-}\right) \leq u_{i}\left(t, \cdot ; u_{0}\right) \leq u_{i}(t, \cdot ; M) \leq M \quad \forall t \geq 0
$$

By (1), Lemmas 4.4 and 4.5, and Dini's Theorem,

$$
u_{i}\left(t, x ; \delta u_{i}^{-}\right)<u_{i}^{*}(x)<u_{i}(t, x ; M) \quad \forall t>0, x \in \mathcal{H}_{i}
$$

and

$$
\lim _{t \rightarrow \infty} u_{i}\left(t, x ; \delta u_{i}^{-}\right)=\lim _{t \rightarrow \infty} u_{i}(t, x ; M)=u_{i}^{*}(x)
$$

uniformly in $x$ on bounded sets. It then follows that

$$
\lim _{t \rightarrow \infty} u_{i}\left(t, x ; u_{0}\right)=u_{i}^{*}(x)
$$

uniformly in $x$ on bounded sets.

We claim that $\left\|u_{i}\left(t, \cdot ; u_{0}\right)-u_{i}^{*}(\cdot)\right\| \rightarrow 0$ as $t \rightarrow \infty$. Assume the claim is not true. Then there are $\epsilon_{0}>0, t_{n} \rightarrow \infty$, and $x_{n}$ with $\left\|x_{n}\right\| \rightarrow \infty$ such that

$$
\left|u_{i}\left(t_{n}, x_{n} ; u_{0}\right)-u_{i}^{*}\left(x_{n}\right)\right| \geq \epsilon_{0} \quad \forall n \in \mathbb{N} .
$$

Then by Lemma 4.3,

$$
\left|u_{i}\left(t_{n}, x_{n} ; u_{0}\right)-u_{i}^{0}\right| \geq \frac{\epsilon_{0}}{2} \quad \forall n \gg 1 .
$$

Let $\tilde{\delta}>0$ and $\tilde{M}>0$ be such that

$$
\tilde{\delta} \leq u_{i}\left(t, \cdot ; u_{0}\right) \leq \tilde{M} \quad \forall t \geq 0 .
$$

For any $\epsilon>0$, let $T>0$ be such that

$$
\left|u_{i}\left(T, \cdot ; \tilde{\delta}, f_{i}^{0}(\cdot)\right)-u_{i}^{0}\right|<\epsilon, \quad\left|u_{i}\left(T, \cdot ; \tilde{M}, f_{i}^{0}(\cdot)\right)-u_{i}^{0}\right|<\epsilon .
$$


Observe that

$$
\tilde{\delta} \leq u_{i}\left(t_{n}-T, x_{n}+x ; u_{0}\right) \leq \tilde{M}
$$

and

$u_{i}\left(t_{n}, x_{n}+\cdot ; u_{0}\right)=u_{i}\left(T, x_{n}+\cdot ; u_{i}\left(t_{n}-T, \cdot ; u_{0}\right)\right)=u_{i}\left(T, \cdot ; u_{i}\left(t_{n}-T, \cdot+x_{n} ; u_{0}\right), f_{i}\left(\cdot+x_{n}, \cdot\right)\right)$

for $n \gg 1$. Then

$$
u_{i}\left(T, \cdot ; \tilde{\delta}, f_{i}\left(\cdot+x_{n}\right)\right) \leq u_{i}\left(t_{n}, x_{n}+\cdot ; u_{0}\right) \leq u_{i}\left(T, \cdot ; \tilde{M}, f_{i}\left(\cdot+x_{n}, \cdot\right)\right)
$$

Observe also that $f_{i}\left(x+x_{n}, u\right) \rightarrow f_{i}^{0}(u)$ as $n \rightarrow \infty$ uniformly in $(x, u)$ on bounded sets. Then by Proposition 3.4,

$$
u_{i}\left(T, x ; \tilde{\delta}, f_{i}\left(\cdot+x_{n}, \cdot\right)\right) \rightarrow u_{i}\left(T, x ; \tilde{\delta}, f_{i}^{0}(\cdot)\right)
$$

and

$$
u_{i}\left(T, x ; \tilde{M}, f_{i}\left(\cdot+x_{n}, \cdot\right)\right) \rightarrow u_{i}\left(T, x ; \tilde{M}, f_{i}^{0}(\cdot)\right)
$$

as $n \rightarrow \infty$ uniformly in $x$ on bounded sets. This together with (4.1) implies that

$\left|u_{i}\left(T, 0 ; \tilde{\delta}, f_{i}\left(\cdot+x_{n}, \cdot\right)\right)-u_{i}^{0}\right|<2 \epsilon, \quad\left|u_{i}\left(T, 0 ; \tilde{M}, f_{i}\left(\cdot+x_{n}, \cdot\right)\right)-u_{i}^{0}\right|<2 \epsilon \quad$ for $\quad n \gg 1$

and then by (4.2),

$$
\left|u_{i}\left(t_{n}, x_{n} ; u_{0}\right)-u_{i}^{0}\right|<2 \epsilon \text { for } n \gg 1 \text {. }
$$

Hence $\lim _{n \rightarrow \infty} u_{i}\left(t_{n}, x_{n} ; u_{0}\right)=u_{i}^{0}$, which is a contradiction. Therefore $\| u_{i}\left(t, \cdot ; u_{0}\right)-$ $u_{i}^{*}(\cdot) \| \rightarrow 0$ as $t \rightarrow \infty$.

(3) By Proposition 3.1, for any $u_{0} \in X_{i}^{+} \backslash\{0\}, u_{i}\left(t, x ; u_{0}\right)>0$ for all $t>0$ and $x \in \mathcal{H}_{i}$. Hence for any given $u_{0} \in X_{i}^{+} \backslash\{0\}$, there are $\sigma>0$ and $r>0$ such that $u_{i}\left(1, x ; u_{0}\right) \geq \sigma$ for $x \in \mathcal{H}_{i}$ with $\|x\| \leq r$. Note that $u_{i}\left(t, \cdot ; u_{0}\right)=u_{i}\left(t-1, \cdot ; u_{i}\left(1, \cdot ; u_{0}\right)\right)$ for $t \geq 1$. (3) then follows from Theorem 2.3 (4) (see next section for the proof of Theorem $2.3(4))$.

5. Spatial spreading speeds and proofs of Theorems 2.2 and 2.3 . In this section, we explore the spreading speeds of (1.1), (1.2), and (1.3), and prove Theorems 2.2 and 2.3. Throughout this section, we assume (H1) and (H2).

We first prove two lemmas.

LEMMA 5.1. Let $\xi \in S^{N-1}, c>0,1 \leq i \leq 3$, and $u_{0} \in X_{i}^{+}$be given.

(1) If $\liminf _{x \cdot \xi \leq c t, t \rightarrow \infty} u_{i}\left(t, x ; u_{0}\right)>0$, then for any $0<c^{\prime}<c$,

$$
\limsup _{x \cdot \xi \leq c^{\prime} t, t \rightarrow \infty}\left|u_{i}\left(t, x ; u_{0}\right)-u_{i}^{*}(x)\right|=0 .
$$

(2) If $\liminf |x \cdot \xi| \leq c t, t \rightarrow \infty u_{i}\left(t, x ; u_{0}\right)>0$, then for any $0<c^{\prime}<c$,

$$
\limsup _{|x \cdot \xi| \leq c^{\prime} t, t \rightarrow \infty}\left|u_{i}\left(t, x ; u_{0}\right)-u_{i}^{*}(x)\right|=0 .
$$

(3) If $\liminf \operatorname{in}_{\|x\| c t, t \rightarrow \infty} u_{i}\left(t, x ; u_{0}\right)>0$, then for any $0<c^{\prime}<c$,

$$
\limsup _{\|x\| \leq c^{\prime} t, t \rightarrow \infty}\left|u_{i}\left(t, x ; u_{0}\right)-u_{i}^{*}(x)\right|=0 .
$$


Proof. (1) Suppose that $\liminf _{x \cdot \xi \leq c t, t \rightarrow \infty} u_{i}\left(t, x ; u_{0}\right)>0$. Then there are $\delta$ and $T>0$ such that

$$
u_{i}\left(t, x ; u_{0}\right) \geq \delta \quad \forall(t, x) \in \mathbb{R}^{+} \times \mathcal{H}_{i}, x \cdot \xi \leq c t, t \geq T .
$$

Assume that the conclusion of (1) is not true. Then there are $0<c^{\prime}<c, \epsilon_{0}>0$, $x_{n} \in \mathcal{H}_{i}$, and $t_{n} \in \mathbb{R}^{+}$with $x_{n} \cdot \xi \leq c^{\prime} t_{n}$ and $t_{n} \rightarrow \infty$ such that

$$
\left|u_{i}\left(t_{n}, x_{n} ; u_{0}\right)-u_{i}^{*}\left(x_{n}\right)\right| \geq \epsilon_{0} \quad \forall n \geq 1 .
$$

Without loss of generality, we may assume that $x_{n} \rightarrow x^{*}$ as $n \rightarrow \infty$ in the case that $\left\{\left\|x_{n}\right\|\right\}$ is bounded (this implies that $f_{i}\left(x+x_{n}, u\right) \rightarrow f_{i}\left(x+x^{*}, u\right)$ uniformly in $(x, u)$ in bounded sets) and $f_{i}\left(x+x_{n}, u\right) \rightarrow f_{i}^{0}(u)$ as $n \rightarrow \infty$ uniformly in $(x, u)$ on bounded sets in the case that $\left\{\left\|x_{n}\right\|\right\}$ is unbounded.

Let $\tilde{u}_{0} \in X_{i}^{+}$,

$$
\tilde{u}_{0}(x)=\delta \quad \forall x \in \mathcal{H}_{i} .
$$

By Theorem 2.1, there is $\tilde{T}>0$ such that

$$
\begin{gathered}
\left|u_{i}\left(\tilde{T}, x ; \tilde{u}_{0}\right)-u_{i}^{*}(x)\right|<\epsilon_{0} \quad \forall x \in \mathcal{H}_{i}, \\
\left|u_{i}\left(\tilde{T}, x ; \tilde{u}_{0}, f_{i}\left(\cdot+x^{*}, \cdot\right)\right)-u_{i}^{*}\left(x+x^{*}\right)\right|<\frac{\epsilon_{0}}{2},
\end{gathered}
$$

and

$$
\left|u_{i}\left(\tilde{T}, x ; \tilde{u}_{0}, f_{i}^{0}\right)-u_{i}^{0}\right|<\frac{\epsilon_{0}}{2} .
$$

Without loss of generality, we may assume that $t_{n}-\tilde{T} \geq T$ for $n \geq 1$. Let $\tilde{u}_{0 n} \in X_{i}^{+}$be such that $\tilde{u}_{0 n}(x)=\delta$ for $x \cdot \xi \leq \frac{c^{\prime}+c}{2}\left(t_{n}-\tilde{T}\right), 0 \leq \tilde{u}_{0 n}(x) \leq \delta$ for $\frac{c^{\prime}+c}{2}\left(t_{n}-\tilde{T}\right) \leq x \cdot \xi \leq c\left(t_{n}-\tilde{T}\right)$, and $\tilde{u}_{0 n}(x)=0$ for $x \cdot \xi \geq c\left(t_{n}-\tilde{T}\right)$. Then

$$
u_{i}\left(t_{n}-\tilde{T}, \cdot ; u_{0}\right) \geq \tilde{u}_{0 n}(\cdot)
$$

and hence

$$
\begin{aligned}
u_{i}\left(t_{n}, x_{n} ; u_{0}\right) & =u_{i}\left(\tilde{T}, x_{n} ; u_{i}\left(t_{n}-\tilde{T}, \cdot ; u_{0}\right)\right) \\
& =u_{i}\left(\tilde{T}, 0 ; u_{i}\left(t_{n}-\tilde{T}, \cdot+x_{n} ; u_{0}\right), f_{i}\left(\cdot+x_{n}, \cdot\right)\right) \\
& \geq u_{i}\left(\tilde{T}, 0 ; \tilde{u}_{0 n}\left(\cdot+x_{n}\right), f_{i}\left(\cdot+x_{n}, \cdot\right)\right) .
\end{aligned}
$$

Observe that $\tilde{u}_{0 n}\left(x+x_{n}\right) \rightarrow \tilde{u}_{0}$ as $n \rightarrow \infty$ uniformly in $x$ on bounded sets. In the case that $f_{i}\left(x+x_{n}, u\right) \rightarrow f_{i}^{0}(u)$, by Proposition 3.4,

$$
u_{i}\left(\tilde{T}, 0 ; \tilde{u}_{0 n}\left(\cdot+x_{n}\right), f_{i}\left(\cdot+x_{n}, \cdot\right)\right) \rightarrow u_{i}\left(\tilde{T}, 0 ; \tilde{u}_{0}, f_{i}^{0}(\cdot)\right)
$$

as $n \rightarrow \infty$. By (5.4) and (5.5),

$$
u_{i}\left(t_{n}, x_{n} ; u_{0}\right)>u_{i}^{0}-\epsilon_{0} / 2 \text { for } n \gg 1 .
$$

By Lemma 4.3,

$$
u_{i}^{0}>u_{i}^{*}\left(x_{n}\right)-\epsilon_{0} / 2 \text { for } n \gg 1 .
$$


By (5.2), (5.6), and (5.7),

$$
\left|u_{i}\left(t_{n}, x_{n} ; u_{0}\right)-u_{i}^{*}\left(x_{n}\right)\right|<\epsilon_{0} \quad \text { for } \quad n \gg 1 .
$$

This contradicts to $(5.1)$.

In the case that $x_{n} \rightarrow x^{*}$, by Proposition 3.4 again,

$$
u_{i}\left(\tilde{T}, 0 ; \tilde{u}_{0 n}\left(\cdot+x_{n}\right), f_{i}\left(\cdot+x_{n}, \cdot\right)\right) \rightarrow u_{i}\left(\tilde{T}, 0 ; \tilde{u}_{0}, f_{i}\left(\cdot+x^{*}, \cdot\right)\right)
$$

as $n \rightarrow \infty$. By (5.3) and (5.5),

$$
u_{i}\left(t_{n}, x_{n} ; u_{0}\right)>u_{i}^{*}\left(x^{*}\right)-\epsilon_{0} / 2 \text { for } n \gg 1 .
$$

By the continuity of $u_{i}^{*}(\cdot)$,

$$
u_{i}^{*}\left(x^{*}\right)>u_{i}^{*}\left(x_{n}\right)-\epsilon_{0} / 2 \text { for } n \gg 1 .
$$

By (5.2), (5.8), and (5.9),

$$
\left|u_{i}\left(t_{n}, x_{n} ; u_{0}\right)-u_{i}^{*}\left(x_{n}\right)\right|<\epsilon_{0} \quad \text { for } n \gg 1 .
$$

This contradicts to (5.1) again.

Hence

$$
\lim _{x \cdot \xi \leq c^{\prime} t, t \rightarrow \infty}\left|u_{i}\left(t, x ; u_{0}\right)-u_{i}^{*}(x)\right|=0
$$

for all $0<c^{\prime}<c$.

(2) It can be proved by the similar arguments as in (1).

(3) It can also be proved by the similar arguments as in (1).

LEMMA 5.2. Let $M>0$ be such that $f_{i}(x, u)<0$ for $x \in \mathcal{H}_{i}, u \geq M$, and $i=1,2,3$. Then for any $\epsilon>0$, there are $p \in \mathbb{N}^{N}$ and $g_{i}: \mathcal{H}_{i} \times \mathbb{R} \rightarrow \mathbb{R}$ such that for any $u \in \mathbb{R}, g_{i}(\cdot, u) \in X_{i, p}, g_{i}(\cdot, \cdot)$ satisfies $(\mathbf{P 1})$ and $(\mathbf{P 3})$, and

$$
\begin{gathered}
f_{i}(x, u) \geq g_{i}(x, u) \quad \forall x \in \mathcal{H}_{i}, u \in[0, M], \\
\hat{g}_{i}(0) \geq f_{i}^{0}(0)-\epsilon
\end{gathered}
$$

where $\hat{g}_{i}(\cdot)$ is as in $(3.21)(i=1,2,3)$.

Proof. By Lemma 4.1, for any $\epsilon>0$, there are $p \in \mathbb{N}^{N}$ and $h_{i}(\cdot) \in X_{i, p} \cap$ $C^{N}\left(\mathcal{H}_{i}, \mathbb{R}\right)$ such that

$$
f_{i}(x, 0) \geq h_{i}(x) \forall x \in \mathcal{H}_{i} \quad \text { and } \quad \hat{h}_{i} \geq f_{i}^{0}(0)-\epsilon
$$

for $i=1,2,3$. Fix $1 \leq i \leq 3$ and choose $M>0$ such that

$$
f_{i}(x, u) \geq h_{i}(x)-M u \quad \text { for } \quad x \in \mathcal{H}_{i}, 0 \leq u \leq M
$$

Let

$$
g_{i}(x, u)=h_{i}(x)-M \quad \forall x \in \mathcal{H}_{i}, u \in \mathbb{R} .
$$

It is not difficult to see that $g_{i}(\cdot, \cdot)(1 \leq i \leq 3)$ satisfy the lemma. 
In the following, $c_{1}^{0}(\xi), c_{2}^{0}(\xi)$, and $c_{3}^{0}(\xi)$ are as in $(2.14),(2.15)$, and $(2.16)$, respectively $\left(\xi \in S^{N-1}\right)$. Observe that $\lambda_{i}\left(\mu, \xi, f_{i}^{0}(0)\right)(i=1,2,3)$ exist and

$$
\left\{\begin{array}{l}
\lambda_{1}\left(\mu, \xi, f_{1}^{0}(0)\right)=f_{1}^{0}(0)+\mu^{2} \\
\lambda_{2}\left(\mu, \xi, f_{2}^{0}(0)\right)=\int_{\mathbb{R}^{N}} e^{-\mu z \cdot \xi} \kappa(z) d z-1+f_{2}^{0}(0) \\
\lambda_{3}\left(\mu, \xi, f_{3}^{0}(0)\right)=\sum_{k \in K} a_{k}\left(e^{-\mu k \cdot \xi}-1\right)+f_{3}^{0}(0) .
\end{array}\right.
$$

If no confusion occurs, we may denote $\lambda_{i}\left(\mu, \xi, f_{i}^{0}(0)\right)$ by $\lambda_{i}(\mu, \xi)(i=1,2,3)$. Observe also that $v_{1}(t, x)=e^{-\mu\left(x \cdot \xi-\frac{\lambda_{1}(\mu, \xi)}{\mu} t\right)}, v_{2}(t, x)=e^{-\mu\left(x \cdot \xi-\frac{\lambda_{2}(\mu, \xi)}{\mu} t\right)}$, and $v_{3}(t, j)=$ $e^{-\mu\left(j \cdot \xi-\frac{\lambda_{3}(\mu, \xi)}{\mu} t\right)}$ are solutions of

$$
\begin{gathered}
v_{t}(t, x)=\Delta v(t, x)+f_{1}^{0}(0) v(t, x), \quad x \in \mathbb{R}^{N}, \\
v_{t}(t, x)=\int_{\mathbb{R}^{N}} \kappa(y-x) v(t, y) d y-v(t, x)+f_{2}^{0}(0) v(t, x), \quad x \in \mathbb{R}^{N},
\end{gathered}
$$

and

$$
v_{t}(t, j)=\sum_{k \in K} a_{k}(v(t, j+k)-v(t, j))+f_{3}^{0}(0) v(t, j), \quad j \in \mathbb{Z}^{N},
$$

respectively.

Proof of Theorem 2.2. Fix $\xi \in S^{N-1}$ and $1 \leq i \leq 3$. We first prove that for any $c^{\prime}>c_{i}^{0}(\xi)$ and $u_{0} \in X_{i}^{+}(\xi)$,

$$
\limsup _{x \cdot \xi \geq c^{\prime} t, t \rightarrow \infty} u_{i}\left(t, x ; u_{0}\right)=0 .
$$

To this end, take a $c$ such that $c^{\prime}>c>c_{i}^{*}(\xi)$. Note that there is $\mu_{i}^{*}>0$ such that

$$
c_{i}^{0}(\xi)=\frac{\lambda_{i}\left(\xi, \mu_{i}^{*}\right)}{\mu_{i}^{*}}
$$

and there is $\mu \in\left(0, \mu_{i}^{*}\right)$ such that

$$
c=\frac{\lambda_{i}(\mu, \xi)}{\mu}
$$

Take $d>M>0$ such that

$$
\begin{gathered}
u_{0}(x) \leq M \quad \text { and } \quad u_{0}(x) \leq d e^{-\mu x \cdot \xi} \quad \forall x \in \mathcal{H}_{i}, \\
f_{i}(x, M)<0 \quad \forall x \in \mathcal{H}_{i}
\end{gathered}
$$

and

$$
f_{i}(x, u)=f_{i}^{0}(u) \quad \text { for } \quad x \cdot \xi \geq-\frac{1}{\mu} \ln \frac{M}{d}(>0) .
$$

Observe that by $(5.14)$ and $(\mathrm{H} 1)$, for $(t, x) \in(0, \infty) \times \mathcal{H}_{i}$ with $d e^{-\mu(x \cdot \xi-c t)} \geq M$, i.e., $x \cdot \xi \leq-\frac{1}{\mu} \ln \frac{M}{d}+c t$,

$$
f_{i}\left(x, d e^{-\mu(x \cdot \xi-c t)}\right)<0<f_{i}^{0}(0) .
$$


By $(5.15)$, for $(t, x) \in(0, \infty) \times \mathcal{H}_{i}$ with $d e^{-\mu(x \cdot \xi-c t)} \leq M$, i.e, $x \cdot \xi \geq-\frac{1}{\mu} \ln \frac{M}{d}+c t$,

$$
f_{i}\left(x, d e^{-\mu(x \cdot \xi-c t)}\right)=f_{i}^{0}\left(d e^{-\mu(x \cdot \xi-c t)}\right) \leq f_{i}^{0}(0) .
$$

It then follows that $u=d e^{-\mu(x \cdot \xi-c t)}$, which is a solution of (5.10) or (5.11) or (5.12) if $i=1$ or 2 or 3 , is a super-solution of (1.i) and hence by Proposition 3.1,

$$
u_{i}\left(t, x ; u_{0}\right) \leq d e^{-\mu(x \cdot \xi-c t)} \quad \forall t>0 x \in \mathcal{H}_{i} .
$$

This implies that (5.13) holds.

Next, we prove that for any $c^{\prime}<c_{i}^{0}(\xi)$ and any $u_{0} \in X_{i}^{+}(\xi)$,

$$
\limsup _{x \cdot \xi \leq c^{\prime} t, t \rightarrow \infty}\left|u_{i}\left(t, x ; u_{0}\right)-u_{i}^{*}(x)\right|=0 .
$$

To this end, take a $c \in \mathbb{R}$ such that $c^{\prime}<c<c_{i}^{0}(\xi)$. Let $M>0$ be such that $u_{0}(x) \leq M$ and $f_{i}(x, M)<0$ for all $x \in \mathcal{H}_{i}$. Then $u \equiv M$ is a super-solution of (1.i) and

$$
u_{i}\left(t, x ; u_{0}\right) \leq M \quad \forall t \geq 0, x \in \mathcal{H}_{i} .
$$

For any $\epsilon>0$, let $g_{i}(\cdot, \cdot)$ be as in Lemma 5.2. By Proposition 3.9, for $\epsilon>0$ sufficiently small,

$$
c_{i}^{*}\left(\xi, g_{i}(\cdot, \cdot)\right) \geq c_{i}^{*}\left(\xi, \hat{g}_{i}(\cdot)\right)>c .
$$

By Propositions 3.1 and 3.8,

$$
\liminf _{x \cdot \xi \leq c t, t \rightarrow \infty} u_{i}\left(t, x ; u_{0}\right) \geq \liminf _{x \cdot \xi \leq c t, t \rightarrow \infty} u_{i}\left(t, x ; u_{0}, g_{i}\right)>0 .
$$

(5.17) then follows from Lemma 5.1.

By (5.13) and (5.17), $c_{i}^{*}(\xi)$ exists and $c_{i}^{*}(\xi)=c_{i}^{0}(\xi)$ for $i=1,2,3$. Moreover, (2.13) holds.

Proof of Theorem 2.3. (1) It can be proved by similar arguments in [64, Theorem $\mathrm{D}(1)$ ]. For completeness, we provide a proof in the following.

Fix $\xi \in S^{N-1}$ and $1 \leq i \leq 3$. Let $u_{0} \in X_{i}^{+}$satisfy that $u_{0}(x)=0$ for $x \in \mathcal{H}_{i}$ with $|x \cdot \xi| \gg 1$. Then there are $u_{0}^{+} \in X_{i}^{+}(\xi)$ and $u_{0}^{-} \in X_{i}^{+}(-\xi)$ such that

$$
u_{0}(x) \leq u_{0}^{ \pm}(x) \quad \forall x \in \mathcal{H}_{i} .
$$

By Proposition 3.1 and Theorem 2.2,

$$
\limsup _{x \cdot \xi \geq c^{\prime} t, t \rightarrow \infty} u_{i}\left(t, x ; u_{0}\right) \leq \limsup _{x \cdot \xi \geq c^{\prime} t, t \rightarrow \infty} u_{i}\left(t, x ; u_{i}^{+}\right)=0 \quad \forall c^{\prime}>c_{i}^{*}(\xi)
$$

and

$$
\limsup _{x \cdot(-\xi) \geq c^{\prime} t, t \rightarrow \infty} u_{i}\left(t, x ; u_{0}\right) \leq \limsup _{x \cdot(-\xi) \geq c^{\prime} t, t \rightarrow \infty} u_{i}\left(t, x ; u_{i}^{-}\right)=0 \quad \forall c^{\prime}>c_{i}^{*}(-\xi)
$$

It then follows that

$$
\limsup _{|x \cdot \xi| \geq c^{\prime} t, t \rightarrow \infty} u_{i}\left(t, x ; u_{0}\right)=0 \quad \forall c^{\prime}>\max \left\{c_{i}^{*}(\xi), c_{i}^{*}(-\xi)\right\} .
$$


(2) Fix $\xi \in S^{N-1}$ and $1 \leq i \leq 3$. For given $0<c^{\prime}<\min \left\{c_{i}^{*}(\xi), c_{i}^{*}(-\xi)\right\}$, take a $c>0$ such that $c^{\prime}<c<\min \left\{c_{i}^{*}(\xi), c_{i}^{*}(-\xi)\right\}$. For given $u_{0} \in X_{i}^{+}$satisfying the condition in Theorem $2.3(2)$, let $M>0$ be such that $u_{0}(x) \leq M$ and $f_{i}(x, M)<0$ for all $x \in \mathcal{H}_{i}$. Then $u \equiv M$ is a super-solution of (1.i) and

$$
u_{i}\left(t, x ; u_{0}\right) \leq M \quad \forall t \geq 0, x \in \mathcal{H}_{i} .
$$

For any $\epsilon>0$, let $g_{i}(\cdot, \cdot)$ be as in Lemma 5.2. By Proposition 3.9, for $\epsilon>0$ sufficiently small,

$$
c_{i}^{*}\left(\xi, g_{i}(\cdot, \cdot)\right) \geq c_{i}^{*}\left(\xi, \hat{g}_{i}(\cdot)\right)>c .
$$

By Propositions 3.1 and 3.8,

$$
\liminf _{|x \cdot \xi| \leq c t, t \rightarrow \infty} u_{i}\left(t, x ; u_{0}\right) \geq \liminf _{|x \cdot \xi| \leq c t, t \rightarrow \infty} u_{i}\left(t, x ; u_{0}, g_{i}\right)>0 .
$$

It then follows from Lemma 5.1 that

$$
\limsup _{|x \cdot \xi| \leq c^{\prime} t, t \rightarrow \infty}\left|u_{i}\left(t, x ; u_{0}\right)-u_{i}^{*}(x)\right|=0 .
$$

(3) It can be proved by similar arguments as in [64, Theorem E (1)]. For completeness again, we provide a proof in the following.

Fix $\xi \in S^{N-1}$ and $1 \leq i \leq 3$. Let $c>\sup _{\xi \in S^{N-1}} c_{i}^{*}(\xi)$. Let $u_{0} \in X_{i}^{+}$be such that $u_{0}(x)=0$ for $\|x\| \gg 1$. Note that for every given $\xi \in S^{N-1}$, there is $\tilde{u}_{0}(\cdot ; \xi) \in X_{i}^{+}(\xi)$ such that $u_{0}(\cdot) \leq \tilde{u}_{0}(\cdot ; \xi)$. By Proposition 3.1,

$$
0 \leq u_{i}\left(t, x ; u_{0}\right) \leq u_{i}\left(t, x ; \tilde{u}_{0}(\cdot ; \xi)\right)
$$

for $t>0$ and $x \in \mathcal{H}_{i}$. It then follows from Theorem 2.2 that

$$
0 \leq \limsup _{x \cdot \xi \geq c t, t \rightarrow \infty} u_{i}\left(t, x ; u_{0}\right) \leq \limsup _{x \cdot \xi \geq c t, t \rightarrow \infty} u_{i}\left(t, x ; \tilde{u}_{0}(\cdot ; \xi)\right)=0
$$

Take any $c^{\prime}>c$. Consider all $x \in \mathcal{H}_{i}$ with $\|x\|=c^{\prime}$. By the compactness of $\partial B\left(0, c^{\prime}\right)=\left\{x \in \mathcal{H}_{i} \mid\|x\|=c^{\prime}\right\}$, there are $\xi_{1}, \xi_{2}, \cdots, \xi_{L} \in S^{N-1}$ such that for every $x \in \partial B\left(0, c^{\prime}\right)$, there is $l(1 \leq l \leq L)$ such that $x \cdot \xi_{l} \geq c$. Hence for every $x \in \mathcal{H}_{i}$ with $\|x\| \geq c^{\prime} t$, there is $1 \leq l \leq L$ such that $x \cdot \xi_{l}=\frac{\|x\|}{c^{\prime}}\left(\frac{c^{\prime}}{\|x\|} x\right) \cdot \xi_{l} \geq \frac{\|x\|}{c^{\prime}} c \geq c t$. By the above arguments,

$$
0 \leq \limsup _{x \cdot \xi_{l} \geq c t, t \rightarrow \infty} u_{i}\left(t, x ; u_{0}\right) \leq \limsup _{x \cdot \xi_{l} \geq c t, t \rightarrow \infty} u_{i}\left(t, x ; \tilde{u}_{0}\left(\cdot ; \xi_{l}\right)\right)=0
$$

for $l=1,2, \cdots L$. This implies that

$$
\limsup _{\|x\| \geq c^{\prime} t, t \rightarrow \infty} u_{i}\left(t, x ; u_{0}\right)=0 .
$$

Since $c^{\prime}>c$ and $c>\sup _{\xi \in S^{N-1}} c_{i}^{*}(\xi)$ are arbitrary, we have that for $c>$ $\sup _{\xi \in S^{N-1}} c_{i}^{*}(\xi)$,

$$
\limsup _{\|x\| \geq c t, t \rightarrow \infty} u_{i}\left(t, x ; u_{0}\right)=0 .
$$


(4) It can be proved by similar arguments as in (2). To be more precise, for given $0<c^{\prime}<\min \left\{c_{i}^{*}(\xi) \mid \xi \in S^{N-1}\right\}$, take a $c>0$ such that $c^{\prime}<c<\min \left\{c_{i}^{*}(\xi) \mid \xi \in\right.$ $\left.S^{N-1}\right\}$. For given $u_{0} \in$ satisfying the condition in Theorem 2.3 (4), let $M>0$ be such that $u_{0}(x) \leq M$ and $f_{i}(x, M)<0$ for all $x \in \mathcal{H}_{i}$. Then $u \equiv M$ is a super-solution of (1.i) and

$$
u_{i}\left(t, x ; u_{0}\right) \leq M \quad \forall t \geq 0, x \in \mathcal{H}_{i} .
$$

For any $\epsilon>0$, let $g_{i}(\cdot, \cdot)$ be as in Lemma 5.2. By Proposition 3.9, for $\epsilon>0$ sufficiently small,

$$
c_{i}^{*}\left(\xi, g_{i}(\cdot, \cdot)\right) \geq c_{i}^{*}\left(\xi, \hat{g}_{i}(\cdot)\right)>c .
$$

By Propositions 3.1 and 3.8,

$$
\liminf _{\|x\| \leq c t, t \rightarrow \infty} u_{i}\left(t, x ; u_{0}\right) \geq \liminf _{\|x\| \leq c t, t \rightarrow \infty} u_{i}\left(t, x ; u_{0}, g_{i}\right)>0 .
$$

It then follows from Lemma 5.1 that

$$
\limsup _{\|x\| \leq c^{\prime} t, t \rightarrow \infty}\left|u_{i}\left(t, x ; u_{0}\right)-u_{i}^{*}(x)\right|=0 .
$$

\section{REFERENCES}

[1] D. G. Aronson and H. F. Weinberger, Nonlinear diffusion in population genetics, combustion, and nerve pulse propagation, in "Partail Differential Equations and Related Topics" (J. Goldstein, Ed.), Lecture Notes in Math., Vol. 466, Springer-Verlag, New York, 1975, pp. $5-49$.

[2] D. G. Aronson and H. F. Weinberger, Multidimensional nonlinear diffusions arising in population genetics, Adv. Math., 30 (1978), pp. 33-76.

[3] P. BAtes And G. ZhaO, Existence, uniqueness and stability of the stationary solution to a nonlocal evolution equation arising in population dispersal, J. Math. Anal. Appl., 332 (2007), pp. 428-440.

[4] H. Berestycki And F. Hamel, Generalized transition waves and their properties, preprint.

[5] H. Berestycki, F. Hamel, And N. Nadirashvili, The speed of propagation for KPP type problems, I - Periodic framework, J. Eur. Math. Soc., 7 (2005), pp. 172-213.

[6] H. Berestycki, F. Hamel, And N. Nadirashvili, The speed of propagation for KPP type problems, II - General domains, J. Amer. Math. Soc., 23 (2010), pp. 1-34.

[7] H. Berestycki, F. Hamel, And L. Roques, Analysis of periodically fragmented environment model: II - Biological invasions and pulsating traveling fronts, J. Math. Pures Appl., 84 (2005), pp. 1101-1146.

[8] H. BerestyCKI, F. HAMEL, AND L. Rossi, Liouville-type results for semilinear elliptic equations in unbounded domains, Ann. Mat. Pura Appl., (4) 186 (2007), pp. 469-507.

[9] R. S. Cantrell and C. Cosner, Spatial Ecology via Reactiond-Diffusion Equations, Series in Mathematical and Computational Biology, John Wiley and Sons, Chichester, UK, 2003.

[10] E. Chasseigne, M. Chaves, And J. D. Rossi, Asymptotic behavior for nonlocal diffusion equations, J. Math. Pures Appl., 86 (2006), pp. 271-291.

[11] X. Chen, S.-C. FU, AND J.-S. GUO, Uniqueness and asymptotics of traveling waves of monostable dynamics on lattices, SIAM J. Math. Anal., 38 (2006), pp. 233-258.

[12] X. Chen And J.-S. Guo, Existence and asymptotic stability of traveling waves of discrete quasilinear monostable equations, J. Diff. Eq., 184 (2002), pp. 549-569.

[13] X. Chen AND J.-S. Guo, Uniqueness and existence of traveling waves for discrete quasilinear monostable dynamics, Math. Ann., 326 (2003), pp. 123-146.

[14] X. Chen, J.-S. Guo, And C.-C. Wu, Traveling waves in discrete periodic media for bistable dynamics, Arch. Ration. Mech. Anal., 189 (2008), pp. 189-236. 
[15] C. Chicone and Y. Latushkin, Evolution Semigroups in Dynamical Systems and Differential Equations, Mathematical Surveys and Monographs, Vol. 70, American Mathematical Society, 1999

[16] J. Clobert, E. Danchin, A. Dhondt, and J. Nichols eds.,Dispersal, Oxford University Press, Oxford, 2001.

[17] C. Cortazar, J. Coville, M. Elgueta and S. Martinez, A nonlocal inhomogeneous dispersal process, J. Differential Equations, 241 (2007), pp. 332-358.

[18] R. Cousens, C. Dytham and R. Law, Dispersal in plants: a population perspective, Oxford University Press, Oxford, 2008.

[19] J. Coville, On a simple criterion for the existence of a principal eigenfunction of some nonlocal operators, J. Diff. Equations, 249 (2010), pp. 2921-2953.

[20] J. Coville And L. Dupaigne, Propagation speed of travelling fronts in non local reactiondiffusion equations, Nonlinear Analysis, 60 (2005), pp. 797-819.

[21] J. Coville, J. DÁvila, And S. Martínez, Existence and uniqueness of solutions to a nonlocal equation with monostable nonlinearity, SIAM J. Math. Anal., 39 (2008), pp. 1693-1709.

[22] P. C. FIfE, Mathematical Aspects of Reacting and Diffusing Systems, Lecture Notes in Biomathematics, 28, Springer-Verlag, Berlin-New York, 1979.

[23] P. C. FIFE, Some nonclassical trends in parabolic and parabolic-like evolutions, in "Trends in nonlinear analysis," pp. 153-191, Springer, Berlin, 2003.

[24] P. C. Fife And L. A. Peletier, Nonlinear diffusion in population genetics, Arch. Rational Mech Anal., 64 (1977), pp. 93-109.

[25] R. Fisher, The wave of advance of advantageous genes, Ann. of Eugenics, 7 (1937), pp. 335369.

[26] M. FREIDLIN AND J. GäRTNER,On the propagation of concentration waves in periodic and ramdom media, Soviet Math. Dokl., 20 (1979), pp. 1282-1286.

[27] M. Grinfeld, G. Hines, V. Hutson, K. Mischaikow, and G. T. Vickers, Non-local dispersal, Differential Integral Equations, 18 (2005), pp. 1299-1320.

[28] J.-S. Guo AND F. HAMEL, Front propagation for discrete periodic monostable equations, Math. Ann., 335 (2006), pp. 489-525.

[29] J.-S. GuO AND C.-H. Wu, Front propagation for a two-dimensional periodic monostable lattice dynamical system, Discrete Contin. Dyn. Syst., 26 (2010), pp. 197-223.

[30] J.-S. GuO AND C.-C. WU, Uniqueness and stability of traveling waves for periodic monostable lattice dynamical system, J. Differential Equations, 246 (2009), pp. 3818-3833.

[31] F. HAMEL, Qualitative properties of monostable pulsating fronts : exponential decay and monotonicity, J. Math. Pures Appl., 89 (2008), pp. 355-399.

[32] D. Henry, Geometric Theory of Semilinear Parabolic Equations, Lecture Notes in Math., 840, Springer-Verlag, Berlin, 1981.

[33] G. Hetzer, W. Shen, And A. Zhang, Effects of spatial variations and dispersal strategies on principal eigenvalues of dispersal operators and spreading speeds of monostable equations, Rocky Mountain Journal of Mathematics, to appear.

[34] J. HuAng And W. Shen, Speeds of spread and propagation for KPP models in time almost and space periodic media, SIAM Journal on Applied Dynamical System, 8 (2009), pp. 790-821.

[35] W. Hudson AND B. ZinNeR, Existence of traveling waves for reaction diffusion equations of Fisher type in periodic media, Boundary value problems for functional-differential equations, pp. 187-199, World Sci. Publ., River Edge, NJ, 1995.

[36] W. Hudson And B. Zinner, Existence of traveling waves for a generalized discrete Fisher's equation, Comm. Appl. Nonlinear Anal., 1 (1994), pp. 23-46.

[37] V. Hutson, S. Martinez, K. Mischaikow, and G. T. Vickers, The evolution of dispersal, J. Math. Biol., 47 (2003), pp. 483-517.

[38] Y. Kametaka, On the nonlinear diffusion equation of Kolmogorov-Petrovskii- Piskunov type, Osaka J. Math., 13 (1976), pp. 11-66.

[39] C.-Y. Kao, Y. Lou, and W. Shen, Random dispersal vs non-Local dispersal, Discrete and Continuous Dynamical Systems, 26 (2010), pp. 551-596

[40] A. Kolmogorov, I. Petrowsky, And N. Piscunov, A study of the equation of diffusion with increase in the quantity of matter, and its application to a biological problem, Bjul. Moskovskogo Gos. Univ., 1 (1937), pp. 1-26.

[41] C. T. Lee, M. F. Hoopes, J. Diehl, W. Gilliland, G. Huxel, E. V. Leaver, K. McCain, J. Umbanhowar And A. Mogilner, Non-local concepts and models in biology, J. theor. Biol., 210 (2001), pp. 201-219.

[42] S. A. Levin, H. C. Muller-Landau, R. Nathan and J. Chave, The ecology and evolution of seed dispersal: a theoretical perspective, Annu. Rev. Eco. Evol. Syst., 34 (2003), pp. 575604 . 
[43] W.-T. LI, Y.-J. SUn, AND Z.-C. WANG, Entire solutions in the Fisher-KPP equation with nonlocal dispersal, Nonlinear Anal. Real World Appl., 11 (2010), pp. 2302-2313.

[44] X. LiAng AND X.-Q. ZhAO, Asymptotic speeds of spread and traveling waves for monotone semiflows with applications, Comm. Pure Appl. Math., 60 (2007), pp. 1-40.

[45] X. LiAng AND X.-Q. ZhaO, Spreading speeds and traveling waves for abstract monostable evolution systems, Journal of Functional Analysis, 259 (2010), pp. 857-903.

[46] X. Liang, Y. Yi, AND X.-Q. ZhaO, Spreading speeds and traveling waves for periodic evolution systems, J. Diff. Eq., 231 (2006), pp. 57-77.

[47] R. Lui, Biological growth and spread modeled by systems of recursions, Math. Biosciences, 93 (1989), pp. 269-312.

[48] N. MAdras, J. WU AND X. Zou, Local-nonlocal interaction and spatial-temporal patterns in single species population over a patchy environment, Canad. Appl. Math. Quart., 4 (1996), pp. 109-134.

[49] A. Mellet, J. Nolen, J.-M. Roquejoffre, and L. Ryzhik, Stability of generalized transition fronts, Comm. Partial Differential Equations, 34 (2009), pp. 521-552.

[50] A. Mellet, J.-M. Roquejoffre And Y. Sire, Generalized fronts for one-dimensional reactiondiffusion equations, Discrete Contin. Dyn. Syst., 26 (2010), pp. 303-312.

[51] J. D. Murray, Mathematical Biology, Springer-Verlag, New York, 1989.

[52] G. Nadin, Traveling fronts in space-time periodic media, J. Math. Pures Appl., (9) 92 (2009), pp. 232-262.

[53] P. Meyer-Nieberg, Banach Lattices, Springer-Verlag, 1991.

[54] J. Nolen, M. Rudd, AND J. XIN, Existence of KPP fronts in spatially-temporally periodic adevction and variational principle for propagation speeds, Dynamics of PDE, 2 (2005), pp. $1-24$.

[55] J. Nolen AND J. XIN, Existence of KPP type fronts in space-time periodic shear flows and a study of minimal speeds based on variational principle, Discrete and Continuous Dynamical Systems, 13 (2005), pp. 1217-1234.

[56] J. Nolen, J.-M. Roquejoffre, L. Ryzhik, and A. Zlato, Existence and non-existence of Fisher-KPP transition fronts, Arch. Ration. Mech. Anal., 203 (2012), pp. 217-246.

[57] A. PAZY, Semigroups of Linear Operators and Applications to Partial Differential Equations, Springer-Verlag New York Berlin Heidelberg Tokyo, 1983.

[58] D. H. SATtinger, On the stability of waves of nonlinear parabolic systems, Advances in Math., 22 (1976), pp. 312-355.

[59] W. Shen, Traveling waves in diffusive random media, J. Dynam. Diff. Equations, 16 (2004), pp. 1011-1060.

[60] W. SHEN, Variational principle for spatial spreading speeds and generalized propagating speeds in time almost periodic and space periodic KPP models, Transactions of the American Mathematical Society, 362 (2010), pp. 5125-5168.

[61] W. SHEN, Spreading and generalized propagating speeds of discrete KPP models in time varying environments, Frontiers of Mathematics in China, 4:3 (2009), pp. 523-562.

[62] W. SHEN, Existence, uniqueness, and stability of generalized traveling waves in time dependent monostable equations, Journal of Dynamics and Differential Equations, 23 (2011), pp.1-44.

[63] W. SHEN, Existence of generalized traveling waves in time recurrent and space periodic monostable equations, Journal of Applied Analysis and Computation, 1 (2011), pp. 69-94.

[64] W. Shen And A. Zhang, Spreading speeds for monostable equations with nonlocal dispersal in space periodic habitats, Journal of Differential Equations, 249 (2010), pp. 749-795.

[65] W. SHen AND A. ZHANG, Stationary solutions and spreading speeds of nonlocal monostable equations in space periodic habitats, Proceedings of the American Mathematical Society, 140 (2012), pp. 1681-1696.

[66] W. Shen And A. Zhang, Traveling wave solutions of spatially periodic nonlocal monostable equations, Communications on Applied Nonlinear Analysis, to appear.

[67] N. Shigesada and K. Kawasaki, Biological Invasions: Theory and Practice, Oxford University Press, 1997.

[68] B. Shorrocks And I. R. Swingland, Living in a Patch Environment, Oxford University Press, New York, 1990.

[69] J. G. Skellam, Random dispersal in theoretical populations, Biometrika, 38 (1951), pp. 196218.

[70] K. UCHIYAMA, The behavior of solutions of some nonlinear diffusion equations for large time, J. Math. Kyoto Univ., 18:3 (1978), pp. 453-508.

[71] H. F. Weinberger, Long-time behavior of a class of biology models, SIAM J. Math. Anal., 13 (1982), pp. 353-396.

[72] H. F. WEINBERGER, On spreading speeds and traveling waves for growth and migration models 
in a periodic habitat, J. Math. Biol., 45 (2002), pp. 511-548.

[73] J. WU AND X. Zou, Asymptotic and periodic boundary value problems of mixed FDEs and wave solutions of lattice differential equations, J. Differential Equations, 135 (1997), pp. 315-357.

[74] B. Zinner, G. Harris, AND W. Hudson, Traveling wavefronts for the discrete Fisher's equation, J. Diff. Eq., 105 (1993), pp. 46-62.

[75] X.-Q. ZhaO, Global attractivity and stability in some monotone discrete dynamical systems, Bull. Austral. Math. Soc., 53 (1996), pp. 305-324.

[76] X.-Q. Zhao, Dynamical Systems in Population Biology, CMS Books in Mathematics, 16, Springer-Verlag, New York, 2003. 